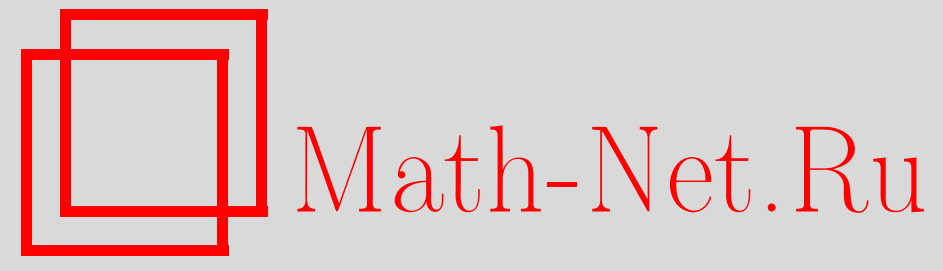

Ш. Сонг, Случайный момент с дифференцируемой функцией условного распределения, Теория вероятн. и ее примен., 2015, том 60, выпуск 4, 740-769

DOI: https://doi.org/10.4213/tvp5033

Использование Общероссийского математического портала Math-Net.Ru подразумевает, что вы прочитали и согласны с пользовательским соглашением http://www.mathnet.ru/rus/agreement

Параметры загрузки:

IP: 54.198 .55 .26

26 апреля 2023 г., 02:26:22

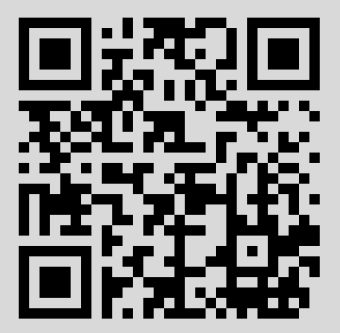




\title{
СЛУЧАЙНЫЙ МОМЕНТ С ДИФФЕРЕНЦИРУЕМОЙ ФУНКЦИЕЙ УСЛОВНОГО РАСПРЕДЕЛЕНИЯ
}

\author{
(Перевод Белкиной Т. А.)
}

\begin{abstract}
В статье приводится общее исследование дифференцируемых моментов, т.е. случайных моментов, функции условных распределений которых дифференцируемы относительно некоторых неубывающих процессов. Класс случайных моментов образует расширение класса начальных моментов. Мы предлагаем подход, основанный на вспомогательном произведении пространств, и устанавливаем фундаментальное соотношение между классами дифференцируемых моментов и мер Кокса. Основываясь на этом соотношении, мы доказываем, что для дифференцируемых моментов сохраняется большинство свойств начальных моментов, в частности, формула условного математического ожидания, формула опционального расщепления и формула расширения фильтрации. Приводится важный пример дифференцируемого момента, иллюстрирующий недостаточность моделей с начальными моментами для приложений.

Ключевые слова и фразы: прогрессивное расширение фильтрации, мера Кокса, формула опционального расщепления, супермартингал Азема.
\end{abstract}

1. Введение. В статье [12] был введен частный класс однодефолтных (one-default) моделей рынка, где моменты дефолта $\tau$ определяются с помощью стохастических дифференциальных уравнений, которым удовлетворяют мартингалы $t \in \mathbb{R}_{+} \rightarrow \mathbb{Q}\left[\tau \leqslant u \mid \mathscr{F}_{t}\right]$ (для каждого фиксированного $u$ ). Это полезный класс случайных моментов, так как они определяются в соответствии с динамикой рынка через стохастические дифференциальные уравнения. Позднее в [23] для этого класса изучались их функции условного распределения $u \rightarrow \mathbb{Q}\left[\tau \leqslant u \mid \mathscr{F}_{t}\right]$ (для каждого фиксированного $t)$. Показано, что случайные моменты $\tau$ из этого класса могут иметь функции условного распределения, дифференцируемые относительно согласованного неубывающего процесса $A$. Будем называть случайные моменты, имеющие дифференцируемую функцию

${ }^{*}$ Laboratoire Analyse et Probabilités, Université d'Evry Val D'Essonne, France; e-mail: shiqi.song@univ-evry.fr

1) Это исследование было поддержано «Chair Markets in Transition» Французской федерации банков. 
условного распределения, дифференцируемыми моментами. Заметим, что начальные моменты (ср. с [10]) являются дифференцируемыми, в то время как результат [23], касающийся динамически определяемых моментов дефолта, показывает, что класс дифференцируемых моментов является важным и очень полезным расширением класса начальных моментов.

Понятие начального момента широко используется в практике моделирования риска дефолта. Этот факт послужил мотивацией для общего исследования более широкого класса дифференцируемых моментов. Цель данной статьи предоставить теоретический базис для понятия дифференцируемых моментов и исследовать их свойства, связанные с приложениями. Заметим, что некоторые из свойств дифференцируемых моментов изучались в [16]. Отметим также, что в настоящей работе и в [16] используются различные подходы. Действительно, для нас центральное место в изучении дифференцируемых моментов - это их соотношение с временной моделью Кокса (так же как начальный момент связан с моментами независимых наблюдений). Данная работа выстроена вокруг этой связи.

Опишем основные моменты данной работы. Используемый здесь подход предполагает, что мера Кокса определена на рассматриваемом стохастическом базисе. Это, тем не менее, может быть не выполнено в общем случае. Стандартный путь (ср. с [20], [22]) преодоления этой технической сложности состоит во вложении первоначального стохастического базиса во вспомогательное вероятностное пространство, на котором конструкция меры Кокса становится возможной. Это проделано в разд. 3. (См. [2] о вложениях вероятностных пространств.) Дифференцируемость случайного момента формально определяется в разд. 4 с помощью понятия семейства процессов і $M$. Первый из основных результатов доказан в теореме 4.2, устанавливающей регулярную версию функции плотности и ставящей меры Кокса в соответствие дифференцируемым моментам.

В соответствии с теоремой 4.2, чтобы получить модель дифференцируемого момента дефолта, достаточно зафиксировать меру Кокса наряду с функцией плотности. Тем не менее, это не только способ обеспечить дифференцируемость случайного момента. Достоинство такого представления состоит в том, что случайный момент определяется для некоторого специального случая, чтобы потом доказать дифференцируемость. Хороший пример такой ситуации приводится в разд. 5, где описан результат [23] о случайных моментах, определенных динамически с помощью стохастических дифференциальных уравнений и являющихся дифференцируемыми.

Интересная особенность моделей риска дефолта заключается в богатстве структуры, подходящей для описания различных ситуаций и 
проведения расчетов. Результат разд. 5 показывает, как класс дифференцируемых моментов может быть общим. Начиная с разд. 7, устанавливаются три формулы для дифференцируемых моментов, а именно: формула условного математического ожидания, формула опционального расщепления и формула расширения фильтрации, причем все они применимы для вычислительных целей. Нужно отметить, что использование временной модели Кокса на вспомогательном пространстве позволяет нам осуществить быстрое и наглядное доказательство этих формул.

2. Вспомогательные результаты. В этом разделе собраны некоторые общие результаты, полезные для дальнейшего.

2.1. Некоторые свойства $\sigma$-алгебр. Напомним некоторые факты о $\sigma$-алгебрах. Пусть $E-$ некоторое множество и $\mathscr{T}-\sigma$-алгебра на $E$. Для $A \subset E$ определим

$$
A \cap \mathscr{T}=\{B \subset A: \exists C \in \mathscr{T}, B=A \cap C\} .
$$

Семейство $A \cap \mathscr{T}$ может быть использовано как семейство подмножеств в $E$ или как $\sigma$-алгебра на $A$. Заметим, что если $f$ и $g$ - два отображения на $E$, такие, что $f=g$ на $A$, то верно $A \cap \sigma(f)=A \cap \sigma(g)$. Нам понадобится еще один факт о $\sigma$-алгебрах. Пусть $\mathscr{T}_{n}, n \in \mathbb{N}^{*},-$ убывающая последовательность $\sigma$-алгебр на $E$. Пусть $F-$ некоторое другое пространство и $\eta$ - некоторое отображение из $F$ в $E$. Тогда

$$
\eta^{-1}\left(\cap_{n \in \mathbb{N}^{*}} \mathscr{T}_{n}\right)=\cap_{n \in \mathbb{N}^{*}} \eta^{-1}\left(\mathscr{T}_{n}\right) .
$$

Очевидно, множество, стоящее в правой части равенства, содержит множество в левой части. Пусть $B-$ некоторый элемент в $\cap_{n \in \mathbb{N} *} \eta^{-1}\left(\mathscr{T}_{n}\right)$. Для любого $n \in \mathbb{N}^{*}$ существует $C_{n} \in \mathscr{T}_{n}$, такое, что $B=\eta^{-1}\left(C_{n}\right)$. Пусть $C=\limsup _{n \rightarrow \infty} C_{n} \in \cap_{n \in \mathbb{N}^{*}} \mathscr{T}_{n}$. Тогда

$$
\eta^{-1}(C)=\eta^{-1}\left(\cap_{n \geqslant 1} \cup_{m \geqslant n} C_{m}\right)=\cap_{n \geqslant 1} \cup_{m \geqslant n} \eta^{-1}\left(C_{m}\right)=B .
$$

Это доказывает равенство, приведенное выше. Рассмотрим теперь подмножество $D$ множества $F$. Применяя предыдущий результат для тождественного отображения из $D$ в $F$, приходим к соотношению в более общей форме.

\section{Лемма 2.1. Имеет место равенство}

$$
D \cap \eta^{-1}\left(\cap_{n \in \mathbb{N}^{*}} \mathscr{T}_{n}\right)=D \cap\left(\cap_{n \in \mathbb{N}^{*}} \eta^{-1}\left(\mathscr{T}_{n}\right)\right)=\cap_{n \in \mathbb{N}^{*}}\left(D \cap \eta^{-1}\left(\mathscr{T}_{n}\right)\right) .
$$

2.2. Пренебрежимые множества. Нам понадобится следующая лемма, которая описывает пополнение $\sigma$-алгебры в терминах самой $\sigma$ алгебры. Это описание будет полезно при сравнении пополнения $\sigma$ алгебры на исходном пространстве с пополнением $\sigma$-алгебры на вспомогательном пространстве (см. лемму 3.1). 
Лемма 2.2. Пусть $\mathscr{T}_{1}, \mathscr{T}_{2}-$ две $\sigma$-алгебры на некотором общем пространстве $\Omega$. Пусть $\nu$ - вероятностная мера, определенная на этих двух $\sigma$-алгебрах. Пусть $\mathscr{N}-$ семейство $\left(\nu, \mathscr{T}_{2}\right)$ пренебрежимьх множеств. Тогда

$$
\mathscr{T}_{1} \vee \sigma(\mathscr{N})=\left\{X \subset \Omega: \exists B \in \mathscr{T}_{1}, A \in \mathscr{T}_{2}, \nu[A]=1, X \cap A=B \cap A\right\} .
$$

Д о к а з а т е л ь с т в. Обозначим правую часть приведенной выше формулы через $\mathscr{J}$. Тогда $\Omega \in \mathscr{J}$. Если $X \in \mathscr{J}$, пусть $B \in \mathscr{T}_{1}$ и $A \in \mathscr{T}_{2}$ таковы, что $\nu[A]=1$ и $X \cap A=B \cap A$. Тогда $X^{c} \cap A=B^{c} \cap A$, т.е. $X^{c} \in \mathscr{J}$. Если $X_{n} \in \mathscr{J}$ для $n \in \mathbb{N}^{*}$, пусть $B_{n} \in \mathscr{T}_{1}$ и $A_{n} \in \mathscr{T}_{2}$ таковы, что $\nu\left[A_{n}\right]=1$ и $X \cap A_{n}=B_{n} \cap A_{n}$. Положим $A=\cap_{n \in \mathbb{N}^{*}} A_{n}$. Тогда $\nu[A]=1-\nu\left[A^{c}\right]=1$, в то время как

$$
\left.\left(\cup_{n \in \mathbb{N}^{*}} X_{n}\right) \cap A=\cup_{n \in \mathbb{N}^{*}}\left(X_{n} \cap A\right)=\cup_{n \in \mathbb{N}^{*}}\left(B_{n} \cap A\right)=\left(\cup_{n \in \mathbb{N}^{*}} B_{n}\right) \cap A\right),
$$

т.е. $\cup_{n \in \mathbb{N}^{*}} X_{n} \in \mathscr{J}$. Семейство $\mathscr{J}$ является $\sigma$-алгеброй.

$\sigma$-алгебра $\mathscr{J}$ содержит, очевидно, $\mathscr{T}_{1}$. Она содержит также $\mathscr{N}$. Для любого $X \in \mathscr{N}$ существует $C \in \mathscr{T}_{2}$, такое, что $\nu[C]=0$ и $X \subset C$. Пусть $A=C^{c}$, тогда $\nu[A]=1$ и $X \cap A=\varnothing=\varnothing \cap A$. Это означает, что $X \in \mathscr{J}$.

С другой стороны, для любого $X \in \mathscr{J}$, пусть $B \in \mathscr{T}_{1}$ и $A \in \mathscr{T}_{2}$, такие, что $\nu[A]=1$ и $X \cap A=B \cap A$. Тогда

$$
X=X \cap A+X \cap A^{c}=B \cap A+X \cap A^{c} \in \mathscr{T}_{1} \vee \sigma(\mathscr{N}) .
$$

Это означает, что $\mathscr{J} \subset \mathscr{T}_{1} \vee \sigma(\mathscr{N})$. Лемма 2.2 доказана.

2.3. Первый нуль неотрицательного супермартингала. Нам понадобится лемма о неотрицательных супермартингалах.

Лемма 2.3. Пусть $Y$ - неотричательный супермартингал, определенный на некотором вероятностном пространстве с фильтраиией. Пусть $X$ - локально ограниченный семимартингал. Рассмотрим предсказуемую скобку $\langle X, Y\rangle$. Тогда

$$
\int_{0}^{\infty} \mathbb{I}_{\left\{Y_{s-}=0\right\}} d\langle X, Y\rangle_{s}=0 .
$$

Пусть $V$ - прочесс, соответствующий сносу $Y$ в его каноническом разложении ( $V$ - прочесс, убьваюоий и равный нулю в нулевой момент). Пусть $R=\inf \left\{t \in \mathbb{R}_{+}: Y_{t}=0\right\}$ u $R^{\prime}=R \mathbb{I}_{\left\{0<R<\infty, Y_{R-}=0\right\}}+$ $\infty \mathbb{I}_{\left\{Y_{R-}>0\right\}}$. Тогда $R^{\prime}$ предсказуем $и \Delta_{R^{\prime}} V=0$ на множестве $\left\{R^{\prime}<\infty\right\}$.

Д о к а з а т е ль с т в о. Пусть $\mathscr{F}=\inf \left\{0<R<\infty, Y_{R-}=0\right\}$. В соответствии с утверждением [8, теорема 2.62, следствие 12.5] (см. также [8, теорема 3.35]),

$$
\left\{Y_{-}=0\right\} \cap(0, \infty)=\mathscr{F} \cap[R, \infty)+\mathscr{F}^{c} \cap(R, \infty) .
$$


Следовательно,

$$
\left[R^{\prime}\right]=(0, R] \cap\left\{Y_{-}=0\right\} \cap(0, \infty)
$$

- предсказуемое множество, т.е. $R^{\prime}$ - предсказуемый момент остановки. Далее имеем

$$
\begin{aligned}
& \int_{0}^{\infty} \mathbb{I}_{\left\{Y_{s-}\right.}=0\} \\
&\left.=\Delta_{R^{\prime}}\langle X, Y\rangle\right\rangle_{s}=\int_{0}^{\infty} \mathbb{I}_{\{0<s \leqslant R\}} \mathbb{I}_{\left\{Y_{s-}<\infty\right\}}=\mathbb{E}\left[\Delta_{R^{\prime}} X \Delta_{R^{\prime}} Y|X, Y\rangle_{s}\right. \\
&\left.\mathscr{F}_{R^{\prime}}\right] \mathbb{I}_{\left\{R^{\prime}<\infty\right\}}=0,
\end{aligned}
$$

что доказывает первую часть леммы.

Что касается второй части леммы, заметим, что $Y_{-}={ }^{p}(Y)-\Delta V$ (где верхний индекс ${ }^{p}$ соответствует обозначению предсказуемой проекции). Следовательно, $Y_{s-}>0$ при $0 \leqslant s<\infty$, если $\Delta_{s} V<0$. Но $Y_{R^{\prime}-}=0$ на множестве $\left\{R^{\prime}<\infty\right\}$, так что $\Delta_{R^{\prime}} V=0$ на $\left\{R^{\prime}<\infty\right\}$. Лемма 2.3 доказана.

2.4. Замена переменной. Пусть $a$ - вещественная неотрицательная непрерывная справа неубывающая функция на $\mathbb{R}_{+}$. Следуя [8], введем правую обратную к $a$ функцию:

$$
c(s)=\inf \left\{u \in \mathbb{R}_{+}: a(u)>s\right\}, \quad s \in \mathbb{R}_{+} .
$$

Тогда $c$ - неотрицательная непрерывная справа неубывающая функция. Имеем:

$$
\begin{gathered}
\{a(u) \geqslant s\}=\{c(s-) \leqslant u\}, \quad\{a(u-) \leqslant s\}=\{c(s) \geqslant u\}, \\
a(c(s)-) \leqslant s \leqslant a(c(s-)), \quad a(u)=\inf \left\{s \in \mathbb{R}_{+}: c(s)>u\right\} .
\end{gathered}
$$

Для любой неотрицательной борелевской функции $f$ справедливо равенство

$$
\int_{[0, \infty)} f(u) d a(u)=\int_{[0, \infty)} \mathbb{I}_{\{c(s-)<\infty\}} f(c(s-)) d s .
$$

В частности, для любого $t \in \mathbb{R}_{+}$

$$
\begin{aligned}
\int_{[0, t]} e^{-a(u)} d a(u) & =\int_{[0, \infty)} \mathbb{I}_{\{c(s-) \leqslant t\}} e^{-a(c(s-))} d s \leqslant \int_{[0, \infty)} \mathbb{I}_{\{s \leqslant a(t)\}} e^{-s} d s \\
& =1-e^{-a(t)}<1 .
\end{aligned}
$$

\section{3. Вспомогательное вероятностное пространство, ассоции- рованное со случайным моментом.}

3.1. Основные элементы постановки задачи. Базовые элементы постановки в данной работе - это стохастический базис $(\Omega, \mathscr{A}, \mathbb{F}, \mathbb{Q})$ и $\tau$, где $(\Omega, \mathscr{A})$ - измеримое пространство, $\mathbb{Q}$ - вероятностная мера на $\mathscr{A}$, и $\mathbb{F}$ - непрерывная справа фильтрация, $\mathbb{F}=\left(\mathscr{F}_{t}\right)_{t \in \mathbb{R}_{+}}$ 
$\left(\mathscr{F}_{\infty}=\vee_{t \in \mathbb{R}_{+}} \mathscr{F}_{t} \subset \mathscr{A}\right)$, содержащая $\left(\mathbb{Q}, \mathscr{F}_{\infty}\right)$-нулевые множества, и, наконец, $\tau$ - случайная величина, принимающая значения в $[0, \infty]$ (и называемая ниже случайным моментом).

Обозначим $\nmid$ отображение из $[0, \infty]^{2}$ в $[0, \infty]$, такое, что для $a, b \in$ $[0, \infty], a \nmid b=a$, если $a \leqslant b$ и $a \nmid b=\infty$, если $a>b$. Введем теперь фильтрацию $\mathbb{G}^{0}=\left(\mathscr{G}_{t}^{0}\right)_{t \in \mathbb{R}_{+}}$, определенную соотношением $\mathscr{G}_{t}^{0}=\cap_{s>t}\left(\mathscr{F}_{s} \vee \sigma(\tau \nmid s)\right)$, и ее пополнение $\mathbb{G}=\left(\mathscr{G}_{t}\right)_{t \in \mathbb{R}_{+}}$, где $\sigma$-алгебра $\mathscr{G}_{t}$ есть $\sigma$-алгебра $\mathscr{G}_{t}^{0}$, пополненная $\left(\mathbb{Q}, \mathscr{F}_{\infty} \vee \sigma(\tau)\right)$-нулевыми множествами. Фильтрация $\mathbb{G}$ является прогрессивным расширением фильтрации $\mathbb{F}$, задаваемым случайным моментом $\tau$.

\section{2. Ассоциированное вспомогательное произведение про-} странств. Определим отображение $\phi(\omega)=(\omega, \tau(\omega))$ из $\Omega$ в произведение пространства $\Omega \times[0, \infty]$ и вспомогательного пространства как произведение пространств $\Omega \times[0, \infty]$, снабженное произведением $\sigma$-алгебр $\mathscr{A} \otimes \mathscr{B}[0, \infty]$ и вероятностной мерой $\check{\mathbb{Q}}[B]=\mathbb{Q}\left[G^{-1}(B)\right], B \in \mathscr{A} \otimes \mathscr{B}[0, \infty]$. Далее, введем отображения

$$
\pi(\omega, u)=\omega, \quad \widehat{\tau}(\omega, u)=u, \quad(\omega, u) \in \Omega \times[0, \infty] .
$$

C помощью отображения $\pi$ переводим фильтрацию $\mathbb{F}$ в определенную в произведении пространств $\Omega \times[0, \infty]$ следующим образом:

$$
\check{\mathbb{F}}=\left(\check{\mathscr{F}}_{t}: t \geqslant 0\right)=\pi^{-1}(\mathbb{F})=\left(\pi^{-1}\left(\mathscr{F}_{t}\right): t \geqslant 0\right) .
$$

При этом для любого $B \in \mathscr{F}_{\infty}$ при $a \in[0, \infty]$ имеет место соотношение $\pi^{-1}(B) \in \check{\mathscr{F}}_{\infty}$, и

$$
\mathbb{Q}[B \cap\{\tau \leqslant a\}]=\check{\mathbb{Q}}\left[\pi^{-1}(B) \cap\{\widehat{\tau} \leqslant a\}\right] .
$$

$\mathrm{C}$ помощью фильтрации $\check{\mathbb{F}}$ и случайного момента $\widehat{\tau}$ определим на произведении пространств начальное расширение и прогрессивное расширение, т.е. $\check{\mathbb{G}}^{0}=\left(\check{\mathscr{G}}_{t}^{0}\right)_{t \geqslant 0}$, определяемую соотношениями $\check{\mathscr{G}}_{t}^{0}=\cap_{s>t}\left(\check{\mathscr{F}}_{s} \vee \sigma(\widehat{\tau} \nmid s)\right)$, и $\check{\mathfrak{G}}=\left(\check{\mathscr{G}}_{t}\right)_{t \geqslant 0}$, где $\check{\mathscr{G}}_{t}$ есть пополнение $\check{\mathscr{G}}_{t}^{0} \check{\mathbb{Q}}$-нулевыми множествами относительно $\check{\mathscr{F}}_{\infty} \vee \sigma(\widehat{\tau})$. Таким образом мы построили квадруплет $(\check{\mathbb{Q}}, \check{\mathbb{F}}, \check{\mathscr{G}}, \check{\tau})$ на произведении пространств на основе исходного квадруплета (дублирующий квадруплет) $(\mathbb{Q}, \mathbb{F}, \mathbb{G}, \tau)$. Таким же путем для любого отображения $X$, определенного на пространстве $\Omega$, определим отображение $\check{X}=X(\pi)$ на произведении пространств.

3 а м е ч а н и е 3.1. Заметим, что существуют две копии вспомогательного произведения пространств, дублирующих случайный момент $\tau$, т.е. отображения $\check{\tau}$ и $\widehat{\tau}$. Эти два представителя неразличимы по мере Ф્. Но если мы хотим построить новую вероятностную меру, такую как мера Кокса, то оперируем только с представителем $\widehat{\tau}$.

3 а м е ч а н и е 3.2. Заметим, что в стохастическом исчислении обычно опускается запись зависимости случайных величин от $\omega$. Следуя этой традиции в данной статье, стохастический процесс $X(t, \omega)$ мы 
обозначаем $X_{t}$, случайную функцию $f(\omega, u)$ записываем в виде $f(u)$ и Т.Д.

3.3. Измеримость соотношения между исходным и вспомогательным пространствами. Сравним теперь различные опциональные $\sigma$-алгебры на исходном и вспомогательном пространствах.

Лемма 3.1. Пусть $\Phi$ - отображение из $\mathbb{R}_{+} \times \Omega$ в $\mathbb{R}_{+} \times(\Omega \times[0, \infty])$, такое, что $\Phi(t, \omega)=(t, \phi(\omega))$. Справедливо следуюшее соотношение:

$$
\mathscr{O}\left(\mathbb{G}^{0}\right)=\Phi^{-1}\left(\mathscr{O}\left(\check{\mathbb{G}}^{0}\right)\right) \subset \Phi^{-1}(\mathscr{O}(\check{\mathbb{G}})) \subset \mathscr{O}(\mathbb{G}) .
$$

Д о к а з а т е л ь с т в о. Имеет место следующее соотношение:

$$
G^{-1}\left(\check{\mathscr{F}}_{t} \vee \sigma(\widehat{\tau} \nmid t)\right)=\mathscr{F}_{t} \vee \sigma(\tau \nmid t), \quad G^{-1}\left(\check{\mathscr{G}}_{t}^{0}\right)=\mathscr{G}_{t}^{0},
$$

для любого $t \in \mathbb{R}_{+}$, и, следовательно, по лемме $2.2, \phi^{-1}\left(\check{\mathscr{G}}_{t}\right) \subset \mathscr{G}_{t}$. Тогда для любого (всюду) càdlàg $\check{G}^{0}$ (соответственно $\left.\breve{G}\right)$-согласованного процесса $X$ процесс $X \circ \Phi$ является càdlàg $\mathbb{G}^{0}$ (соответственно $\mathbb{G}$ )согласованным процессом. Следовательно, $\Phi^{-1}\left(\mathscr{O}\left(\check{\mathbb{G}}^{0}\right)\right) \subset \mathscr{O}\left(\mathbb{G}^{0}\right)$, $\Phi^{-1}(\mathscr{O}(\check{\mathbb{G}})) \subset \mathscr{O}(\mathbb{G})$.

Для любого момента остановки $S$ относительно $\mathbb{G}^{0}$ пусть $X=\mathbb{I}_{[S, \infty)}$. Для $t \in \mathbb{Q}_{+}$пусть $f_{t}-\check{\mathscr{G}}_{t}^{0}$-измеримая функция, ограниченная значениями 0 и 1 , такая, что $X_{t}=f_{t}(\phi)$ (см. [8, теорема 1.5]). Пусть

$$
\bar{S}(\omega, u)=\inf \left\{s \in \mathbb{Q}_{+}: f_{s}(\omega, u)=1\right\} .
$$

$\bar{S}$ является $\check{G}^{0}$-моментом остановки, и $S=\bar{S}(\phi)$, т.е.

$$
X=\left(\mathbb{I}_{[\bar{S}, \infty)}\right) \circ \Phi \in \Phi^{-1}\left(\mathscr{O}\left(\check{\mathbb{G}}^{0}\right)\right) .
$$

Из [8, теорема 3.17], следует, что

$$
\mathscr{O}\left(\mathbb{G}^{0}\right) \subset \Phi^{-1}\left(\mathscr{O}\left(\check{\mathbb{G}}^{0}\right)\right) \subset \Phi^{-1}(\mathscr{O}(\check{\mathbb{G}}))
$$

Лемма 3.1 доказана.

Лемма 3.2. Пусть $а \in \mathbb{R}_{+} u H-$ неотрицательный $\check{G}^{0}$-опииональный процесс. Пусть также $A-\mathbb{F}$-согласованный неубъвающий прочесс. Рассмотрим $H$ как функцию $H_{t}(\omega, u)$ трех переменньг $t \in \mathbb{R}_{+}$, $(\omega, u) \in \Omega \times[0, \infty]$. Тогда процесс $t \rightarrow \int_{[0, a]} H_{t}(v) d A_{v}$ является $\mathbb{F}$ опциональным на интервале $[a, \infty)$.

Д о к а з а т е л ь с т в о. Рассмотрим множество Н ограниченных $\check{G}^{0}$-опциональных процессов $H$, для которых выполнено утверждение леммы. $\mathbf{H}$ - векторное пространство, содержащее постоянные функции и замкнутое в равномерной метрике, т.е. функциональный монотонный класс в смысле [19, теорема 3.2]. Пусть $\mathbf{H}^{0}-$ множество всех ограниченных $\check{G ̆}^{0}$ адаптированных càdlàg процессов. Множество $\mathbf{H}^{0}$ 
замкнуто относительно умножения. Покажем, что любой элемент $H$ из множества $\mathbf{H}^{0}$ является элементом $\mathbf{H}$. Действительно, для фиксированного $t \in \mathbb{R}_{+}$по теореме о монотонных классах случайная величина $\int_{[0, a]} H_{t}(v) d A_{v}$ является $\mathscr{F}_{t+\varepsilon}$-измеримой для любого $\varepsilon>0$, следовательно, она $\mathscr{F}_{t}$-измерима. Кроме того, процесс $\left(\int_{[0, a]} H_{t}(v) d A_{v}, t \in \mathbb{R}_{+}\right)$ является càdlàg процессом по теореме о мажорируемой сходимости. Применяя результат $[19,(3.2)]$, получаем, что Н содержит все ограниченные

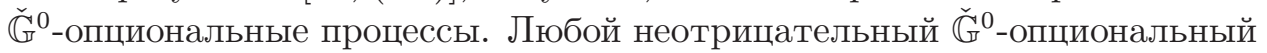
процесс является возрастающим пределом последовательности ограниченных $\check{G}^{0}$-опциональных процессов. Таким образом, лемма 3.2 доказана.

\section{4. Дифференцируемый момент и мера Кокса.}

4.1. Возрастающее семейство мартингалов, ассоциированное со случайным моментом. С вычислительной точки зрения модель риска дефолта на прогрессивном расширении фильтрации характеризуется функцией условного распределения момента дефолта. Это понятие формализовано в [12] с помощью следующего определения.

О п р е д е л е н и е 4.1. i $M(\mathbb{Q}, \mathbb{F})$-семейство (или коротко і $M$-семейство) 一 это семейство процессов $\left(M^{u}: u \in[0, \infty]\right)$, удовлетворяющее следующим условиям:

(1) для каждого $u \in[0, \infty]$ процесс $M^{u}$ является $(\mathbb{Q}, \mathbb{F})$-мартингалом на $[u, \infty]$, принимающем значения на отрезке $[0,1]$;

(2) для каждого $t \in[0, \infty]$ случайное отображение $u \in[0, t] \rightarrow M_{t}^{u}$ является всюду непрерывной справа неубывающей функцией;

(3) $M_{\infty}^{\infty}=1$.

При замене условий (1) и (2) на следующие условия:

$\left(1^{\prime}\right)$ для каждого $u \in[0, \infty]$ процесс $M^{u}$ является $(\mathbb{Q}, \mathbb{F})$-мартингалом на $[0, \infty]$, принимающим значения на отрезке $[0,1]$;

$\left(2^{\prime}\right)$ для каждого $t \in[0, \infty]$ случайное отображение $u \in[0, \infty] \rightarrow M_{t}^{u}$ является всюду непрерывной справа неубывающей функцией; мы будем говорить, что семейство і $M$ полное.

Следующая теорема заимствована из статьи [12].

Теорема 4.1. (i) Для любого случайного момента $\tau$ на стохастическом базисе $(\Omega, \mathscr{A}, \mathbb{F}, \mathbb{Q})$ существует полное і $M$-семейство процессов $\left(M^{u}: u \in[0, \infty]\right)$, такое, что для $u, t \in[0, \infty]$

$$
M_{t}^{u}=\mathbb{Q}\left[\tau \leqslant u \mid \mathscr{F}_{t}\right](\text { почти наверное }) .
$$

Это семейство единственно в том смисле, что, если $\left(\widetilde{M}^{u}: u \in\right.$ $[0, \infty])$ - некоторое другое іМ-семейство, удовлетворяюшее условиям, приведенным выше, то существует $\mathbb{Q}$-нулевое множество $A$, такое, что для $\omega \notin A$ выполнено равенство $M_{t}^{u}(\omega)=\widetilde{M}_{t}^{u}(\omega)$ для всех 
$0 \leqslant u, t \leqslant \infty$. Будем говорить, ито $\left(M^{u}: u \in[0, \infty]\right)$ ассоциировано $c$ триплетом $(\mathbb{Q}, \mathbb{F}, \tau)$.

(ii) Пусть $\left(M^{u}: u \in[0, \infty]\right)$ - некоторое семейство і $M(\mathbb{Q}, \mathbb{F})$. Существует единственная вероятностная мера $\mathbb{Q}^{\prime}$ на вспомогательном

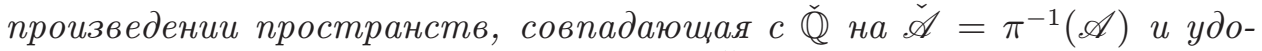
влетворяющая соотношениям $\mathbb{Q}^{\prime}\left[\widehat{\tau} \leqslant u \mid \check{\mathscr{F}}_{t}\right]=M_{t}^{u}(\pi), 0 \leqslant u \leqslant t \leqslant \infty, u$ $\mathbb{Q}^{\prime}[\widehat{\tau} \leqslant u \mid \check{\mathscr{A}}]=M_{\infty}^{u}(\pi)$. Будем говорить, ито мера $\mathbb{Q}^{\prime}$ на вспомогательном произведении пространств ассочиирована с триплетом $(\mathbb{Q}, \mathbb{F}, \mathrm{i} M)$.

(iii) Из (ii) следует, ито любое іМ-семейство имеет полное расширение.

Для оперирования і $M$-семействами нам также понадобятся следующие технические леммы. Первая из них является прямым следствием определения и теоремы о монотонных классах.

Лемма 4.1. Пусть $\left(M^{u}: u \in[0, \infty]\right)$ - iM-семейство, ассоииированное с триплетом $(\mathbb{Q}, \mathbb{F}, \tau)$ из теоремь 4.1. Для любого $t \in[0, \infty]$ и любой неотрииательной $\mathscr{F}_{t} \otimes \mathscr{B}[0, \infty]$-измеримой функиии $f$ имеет место равенство

$$
\mathbb{E}[f(\cdot, \tau)]=\mathbb{E}\left[\int_{[0, \infty]} f(\cdot, u) d_{u} M_{t}^{u}\right]
$$

Лемма 4.2. Для любого полного $\mathrm{i} M(\mathbb{Q}, \mathbb{F})$-семейства $\left(M^{u}: u \in\right.$ $[0, \infty])$ отображения $\left(M_{t}^{t}\right)_{t \in \mathbb{R}_{+}}$определяют $\mathbb{F}$-опциональный процесс.

Д о к а з а т е л ь с т в о. Отображение $((t, \omega), u) \in\left(\mathbb{R}_{+} \times \Omega\right) \times$ $[0, \infty] \rightarrow M_{t}^{u}(\omega)$ является $\mathscr{O}(\mathbb{F}) \times \mathscr{B}[0, \infty]$-измеримым, так как непрерывно справа по $u$. Определим отображение $\psi$ из $\mathbb{R}_{+} \times \Omega$ в $\left(\mathbb{R}_{+} \times \Omega\right) \times[0, \infty]$, такое, что $\psi(t, \omega)=((t, \omega), t)$. Тогда

$$
\psi^{-1}(\mathscr{O}(\mathbb{F}) \times \mathscr{B}[0, \infty])=\mathscr{O}(\mathbb{F}) .
$$

Отсюда следует утверждение леммы 4.2.

Лемма 4.3. Пусть $\mathbf{M}=\left(M^{u}: u \in[0, \infty]\right)-\mathrm{i} M$-семейство, ассоииированное $c(\mathbb{Q}, \mathbb{F}, \tau)$. Пусть $\widehat{\mathbf{M}}=\left(\widehat{M}^{u}: u \in[0, \infty]\right)-\mathrm{i} M$-семейство на вспомогательном произведении пространств, ассочиированное $с$ $(\check{\mathbb{Q}}, \check{\mathbb{F}}, \widehat{\tau})$. Тогда $\mathbf{M}(\pi)$ является версией $\widehat{\mathbf{M}}$, а $\widehat{\mathbf{M}}(\mathscr{G})$ является версией M.

Д о к а з а т е л ь с т в о. Лемма 4.3 является следствием непрерывности справа $\widehat{\mathbf{M}}$ и $\mathbf{M}$, а также следующего равенства. Для $u, t \in[0, \infty]$ при $u \leqslant t$ и для любого $B \in \mathscr{F}_{t}$

$$
\begin{aligned}
\check{\mathbb{E}}\left[\mathbb{I}_{B}(\pi) M_{t}^{u}(\pi)\right] & =\mathbb{E}\left[\mathbb{I}_{B} M_{t}^{u}\right]=\mathbb{E}\left[\mathbb{I}_{B} \mathbb{I}_{\{\tau \leqslant u\}}\right]=\check{\mathbb{E}}\left[\mathbb{I}_{B}(\pi) \mathbb{I}_{\{\widehat{\tau} \leqslant u\}}\right] \\
& =\check{\mathbb{E}}\left[\mathbb{I}_{B}(\pi) \widehat{M}_{t}^{u}\right]=\mathbb{E}\left[\mathbb{I}_{B} \widehat{M}_{t}^{u}(\phi)\right] .
\end{aligned}
$$




\section{2. Дифференцируемое і $M$-семейство.}

О п р е д е л е н и е 4.2. Пусть $\left(M^{u}: u \in[0, \infty]\right)$ - некоторое $\mathrm{i} M(\mathbb{Q}, \mathbb{F})$-семейство и $A-$ некоторый $\mathbb{F}$-адаптированный неотрицательный неубывающий процесс. Для $t \in[0, \infty]$ семейство $\left(M^{u}: u \in[0, \infty]\right)$ называется дифференцируемым относительно $A$ с горизонтом $t$, если существует неотрицательная $\mathscr{F}_{t} \otimes \mathscr{B}[0, \infty]$-измеримая функция $\mathrm{p}_{t}(\omega, v)$, такая, что для почти всех $\omega$

$$
M_{t}^{u}=\int_{[0, u]} \mathrm{p}_{t}(v) d A_{v} \quad \forall u \in[0, t] .
$$

(Как обычно, мы опускаем символ $\omega$. .) Будем называть $\mathrm{p}_{t}$ функцией плотности с горизонтом $t$. Пусть $T \in[0, \infty]$. Если $\left(M^{u}: u \in[0, \infty]\right)$ дифференцируемо при любом горизонте $t \in[0, T)$ относительно одного и того же процесса $A$, будем говорить, что $\left(M^{u}: u \in[0, \infty]\right)$ дифференцируемо на интервале $[0, T)$.

3 а м е ч а н и е 4.1. Если заменить $d A_{v}$ на $e^{-A_{v}} d A_{v}$ и $\mathrm{p}_{t}(v)$ на $\mathrm{p}_{t}(v) e^{A_{v}}$, то в определении можно предположить, что $A_{t}<1$ для $t \in \mathbb{R}_{+}$ (см. разд. 2.4).

Следующая лемма является следствием леммы 4.3.

Лемма 4.4. Пусть $\mathbf{M}=\left(M^{u}: u \in[0, \infty]\right)$ является і $M$-семейством, ассоииированньлм $с(\mathbb{Q}, \mathbb{F}, \tau)$. Пусть $\widehat{\mathbf{M}}=\left(\widehat{M}^{u}: u \in[0, \infty]\right)-$ $\mathrm{i} M$-семейство на вспомогательном произведении пространств, ассоииированное с $(\check{\mathbb{Q}}, \check{\mathbb{F}}, \widehat{\tau})$. Пусть $A-\mathbb{F}$-адаптированный неотрицательный неубыввююий прочесс и $\check{A}=A(\pi)$.

Если $\mathbf{M}$ дифференцируемо относительно $А$ на $[0, T), T \in[0, \infty], c$ функцией плотности $\mathrm{p}$, то $\widehat{\mathbf{M}}$ дифференцируемо относительно $\breve{A}$ на $[0, T)$ с функиией плотности ц́, определяемой соотношением

$$
\check{\mathrm{p}}_{t}((\omega, u), v)=\mathrm{p}_{t}(\pi(\omega, u), v), \quad(\omega, u) \in \Omega \times[0, \infty], \quad v \in[0, \infty] .
$$

Обратно, если $\widehat{\mathbf{M}}$ дифференцируемо относительно $\check{A} н а[0, T)$ с функиией плотности $\widehat{\mathrm{p}}$, то $\mathbf{M}$ дифференцируемо относительно $A$ на $[0, T)$ с функиией плотности р, определяемой равенством

$$
\mathrm{p}_{t}(\omega, v)=\widehat{\mathrm{p}}_{t}(\phi(\omega), v), \quad \omega \in \Omega, \quad v \in[0, \infty] .
$$

4.3. Мера Кокса. Введем понятие меры Кокса (см. дополнительно [5], [12]).

О п р е д е л е н и е 4.3. Вероятностная мера $\mathbb{Q}^{\circ}$ на $\mathscr{F}_{\infty} \vee \sigma(\tau)$ называется мерой Кокса относительно $\left.\mathbb{Q}\right|_{\mathscr{F}_{\infty}}\left(\right.$ сужения $\mathbb{Q}$ на $\left.\mathscr{F}_{\infty}\right)$, если существует $\mathbb{F}$-адаптированный неотрицательный неубывающий процесс $A$, такой, что

(i) $\mathbb{Q}^{\circ}=\mathbb{Q}$ на $\mathscr{F}_{\infty}$; 
(ii) для всех $u, t \in[0, \infty], u \leqslant t$, верно $\mathbb{Q}^{\circ}\left[\tau \leqslant u \mid \mathscr{F}_{t}\right]=A_{u}$.

Будем говорить также, что $\mathbb{Q}^{\circ}$ - мера Кокса, базирующаяся на $A$, ассоциированная с $(\mathbb{Q}, \mathbb{F}, \tau)$.

3 а м е ч а н и е 4.2. Заметим, что і $M$-семейство, ассоциированное с $\left(\mathbb{Q}^{\circ}, \mathbb{F}, \tau\right)$, задается соотношением $M_{t}^{u}=A_{u}, 0 \leqslant u \leqslant t \leqslant \infty$.

Лемма 4.5. Любой $\mathbb{F}$-адаптированный неотрицательный неубывающий процесс $A=\left(A_{t}, t \in \mathbb{R}_{+}\right)$, такой, что $A_{\infty-} \leqslant 1$, может быть расширен на область $[0, \infty]$, если положить $A_{\infty}=1$. Пусть $\check{A}=A(\pi)$. Тогда существует мера Кокса $\check{\mathbb{Q}}^{\circ}$, базирующаяся на $\check{A}$, ассоциированная с $(\check{\mathbb{Q}}, \check{\mathbb{F}}, \widehat{\tau})$ на вспомогательном произведении пространств.

Д о к а з а т е л ь с в о. Достаточно определить меру Кокса равенством

$$
\begin{aligned}
\check{\mathbb{Q}}^{\circ}[h] & =\int_{(\omega, u) \in \Omega \times[0, \infty]} d \check{\mathbb{Q}}(\omega, u) \int_{[0, \infty]} h(\pi(\omega, u), v) d_{v} \check{A}_{v}(\omega, u) \\
& =\int_{\omega \in \Omega} d \mathbb{Q}(\omega) \int_{[0, \infty]} h(\omega, v) d_{v} A_{v}(\omega) \\
& =\mathbb{E}\left[\int_{[0, \infty]} h(\cdot, v) d A_{v}\right]
\end{aligned}
$$

для неотрицательно $\mathscr{F}_{\infty} \otimes \mathscr{B}[0, \infty]$-измеримой функции $h$.

\section{4. Соотношение между дифференцируемыми моментами} и мерами Кокса. Следующая теорема описывает связь дифференцируемых моментов и мер Кокса. Устанавливается регулярная версия функции плотности р, которая обеспечивает основу для дальнейших вычислений в оставшейся части данной работы.

Теорема 4.2. Пусть $Z$ есть $(\mathbb{Q}, \mathbb{F})$-супермартингал вида $\mathbb{Q}[t<$ $\left.\tau \mid \mathscr{F}_{t}\right], t \in \mathbb{R}_{+}($так назььваемьии супермартингал Азема от $\tau)$. Пусть $A-\mathbb{F}$-согласованный отрицательньй неубывающий процесс, такой ито $A_{\infty}=1$ u $A_{t}<1$ для $t \in \mathbb{R}_{+}$. Пусть $\left(M^{u}: u \in[0, \infty]\right)-$ $\mathrm{i} M$-семейство, ассоииированное $c(\mathbb{Q}, \mathbb{F}, \tau)$, которое предполагается дифферениируемьм относительно $A$ на $[0, T)$ при $T \in[0, \infty]$, с функиией плотности р. Пусть $\check{\mathbb{Q}}^{\circ}$ - мера Кокса на вспомогательном произведении пространств, базирующаяся на $\check{A}$, ассочиированная $c(\check{\mathbb{Q}}, \check{\mathbb{F}}, \widehat{\tau})$.

Тогда существует функиия трех переменньх $\mathrm{p}_{t+}(\omega, u),(\omega, u) \in \Omega \times$ $[0, \infty], t \in[0, T)$, такая, ито

(1) процесс $\mathrm{p}_{t+}, t \in[0, T)$, является $\check{G}^{0}$-согласованнылм на интервале $[0, T), и$ для всех $(\omega, u) \in \Omega \times[0, \infty]$ отображение $t \rightarrow \mathrm{p}_{t+}(\omega, u)$ всюду непрерьвно справа и $\check{\mathbb{Q}}^{\circ}$ почти всюду непрерьвно слева на $[0, T)$;

(2) $\check{\mathbb{Q}}$ абсолютно непрерьвна относительно $\check{\mathbb{Q}}^{\circ}$ на $\check{\mathscr{G}}_{t}^{0}$ для каждого $t \in[0, T)$ u проиесс

$$
\mathrm{P}_{t}=\mathbb{I}_{\{t<u\}} \frac{Z_{t}(\omega)}{1-A_{t}(\omega)}+\mathbb{I}_{\{u \leqslant t\}} \mathrm{p}_{t+}(\omega, u), \quad t \in[0, T),
$$


является соответствуюшим прочессом плотности;

(3) $M_{t}^{a} u \int_{[0, a]} \mathrm{p}_{t+}(v) d A_{v}$ (как обиччно, $\omega$ опущено в обозначении р) при $0 \leqslant a \leqslant t<T$ являются $\mathbb{Q}$-неразличимьли как двухпараметрические прочессьи. В частности, $t \rightarrow \int_{[0, a]} \mathrm{p}_{t+}(v) d A_{v}$ является càdlàg прочессом на $[a, T)$.

3 а м е ч а н и е 4.3. Будем использовать обозначение р для оригинальной версии функции плотности и $\mathrm{p}_{+}$— для версии функции плотности, установленной в этой теореме. Позже мы будем рассматривать ее третью версию.

Д о к а з а т е л с т в о т е о р е м ы 4.2. Воспользуемся обозначениями леммы 4.4. Для каждого $t \in[0, T)$, любой неотрицательной ограниченной борелевской функции $h$ на $[0, \infty]$ и любого $B \in \mathscr{F}_{t}$, применяя лемму 4.1, получаем

$$
\begin{aligned}
\check{\mathbb{E}}\left[\mathbb{I}_{B}(\pi) h(\widehat{\tau} \nmid t) \mathbb{I}_{\{\hat{\tau} \leqslant t\}}\right] & =\mathbb{E}\left[\mathbb{I}_{B} h(\tau \nmid t) \mathbb{I}_{\{\tau \leqslant t\}}\right]=\mathbb{E}\left[\mathbb{I}_{B} \int_{[0, t]} h(u) d_{u} M_{t}^{u}\right] \\
& =\mathbb{E}\left[\mathbb{I}_{B} \int_{[0, t]} h(u) \mathbf{p}_{t}(u) d A_{u}\right] \\
& =\check{\mathbb{E}}^{\circ}\left[\mathbb{I}_{B}(\pi) h(\widehat{\tau} \nmid t) \mathbb{I}_{\{\hat{\tau} \leqslant t\}} \mathbf{p}_{t}\right],
\end{aligned}
$$

И

$$
\begin{aligned}
\check{\mathbb{E}}\left[\mathbb{I}_{B}(\pi) h(\widehat{\tau} \nmid t) \mathbb{I}_{\{t<\hat{\tau}\}}\right] & =\mathbb{E}\left[\mathbb{I}_{B} h(\tau \nmid t) \mathbb{I}_{\{t<\tau\}}\right]=\mathbb{E}\left[\mathbb{I}_{B} h(\infty) \mathbb{I}_{\{t<\tau\}}\right] \\
& =\mathbb{E}\left[\mathbb{I}_{B} h(\infty) Z_{t}\right]=\mathbb{E}\left[\mathbb{I}_{B} h(\infty) \frac{Z_{t}}{1-A_{t}}\left(1-A_{t}\right)\right] \\
& =\check{\mathbb{E}}^{\circ}\left[\mathbb{I}_{B}(\pi) h(\infty) \frac{\check{Z}_{t}}{1-\check{A}_{t}} \mathbb{I}_{\{t<\hat{\tau}\}}\right] \\
& =\check{\mathbb{E}}^{\circ}\left[\mathbb{I}_{B}(\pi) h(\widehat{\tau} \nmid t) \frac{\check{Z}_{t}}{1-\check{A}_{t}} \mathbb{I}_{\{t<\hat{\tau}\}}\right] .
\end{aligned}
$$

Из этих двух тождеств следует, что

$$
\check{\mathbb{E}}\left[\mathbb{I}_{B}(\pi) h(\widehat{\tau} \nmid t)\right]=\check{\mathbb{E}} \circ\left[\mathbb{I}_{B}(\pi) h(\widehat{\tau} \nmid t)\left(\mathbb{I}_{\{t<\hat{\tau}\}} \frac{\check{Z}_{t}}{1-\check{A}_{t}}+\mathbb{I}_{\{\hat{\tau} \leqslant t\}} \mathrm{p}_{t}\right)\right] .
$$

Это означает, что $\check{\mathbb{Q}}$ абсолютно непрерывна относительно $\check{\mathbb{Q}}^{\circ}$ на $\check{\mathscr{F}}_{t} \mathrm{~V}$ $\sigma(\widehat{\tau} \nmid t)$ с плотностью

$$
\mathbb{I}_{\{t<u\}} \frac{Z_{t}(\omega)}{1-A_{t}(\omega)}+\mathbb{I}_{\{u \leqslant t\}} \mathrm{p}_{t}(\omega, u)
$$

Приведенный выше процесс является $\check{\mathbb{Q}}^{\circ}$-мартингалом на фильтрации $\mathscr{\mathscr { F }}_{t} \vee \sigma(\widehat{\tau} \nmid t), t \in[0, T)$ (в обычном смысле понятия мартингала). 
По теореме сходимости мартингалов (см. [8, теорема 2.44 (лемма Фелльмера)] или $\left[7\right.$, с. 142]), существует всюду непрерывный справа $\left(\check{\mathbb{Q}}^{\circ}, \check{G}^{0}\right)$ мартингал Р на интервале $[0, T)$, такой, что для $\mathbb{Q}^{\circ}$-почти всех $(\omega, u)$

$$
\mathrm{P}_{t}(\omega, u)=\lim _{s \in \mathbb{Q}_{+}: s \downarrow t}\left(\mathbb{I}_{\{s<u\}} \frac{Z_{s}(\omega)}{1-A_{s}(\omega)}+\mathbb{I}_{\{u \leqslant s\}} \mathrm{p}_{s}(\omega, u)\right) \quad \forall t \in[0, T) .
$$

Кроме того, существует $\check{G}^{0}$-момент остановки $\zeta$, такой, что $\check{\mathbb{Q}}^{\circ}[\zeta<$ $\infty]=0$ и $\mathrm{P}_{-}$существует в каждой точке кроме $\zeta$. Пусть $\mathrm{p}_{t+}(\omega, u)=$ $\mathbb{I}_{\{u \leqslant t\}} \mathrm{P}_{t}(\omega, u)$. Тогда

$$
\mathrm{P}_{t}(\omega, u)=\mathbb{I}_{\{t<u\}} \frac{Z_{t}(\omega)}{1-A_{t}(\omega)}+\mathbb{I}_{\{u \leqslant t\}} \mathrm{p}_{t+}(\omega, u)
$$

является функцией плотности $\check{\mathbb{Q}}$ относительно $\check{\mathbb{Q}}^{\circ}$ на $\check{\mathscr{G}}_{t}^{0}$.

Пусть $S \geqslant a$, где $a \in[0, T),-\mathbb{F}$-момент остановки. При $B \in \mathscr{F}_{S}$

$$
\begin{aligned}
\mathbb{E}\left[\mathbb{I}_{B} \mathbb{I}_{\{S<T\}} M_{S}^{a}\right] & =\mathbb{E}\left[\mathbb{I}_{B} \mathbb{I}_{\{S<T\}} \mathbb{I}_{\{\tau \leqslant a\}}\right]=\check{\mathbb{E}}\left[\mathbb{I}_{B}(\pi) \mathbb{I}_{\{\check{S}<T\}} \mathbb{I}_{\{\hat{\tau} \leqslant a\}}\right] \\
& =\check{\mathbb{E}}^{\circ}\left[\mathbb{I}_{B}(\pi) \mathbb{I}_{\{\check{S}<T\}} \mathbb{I}_{\{\hat{\tau} \leqslant a\}} \mathrm{p}_{\check{S}+}\right] \\
& =\mathbb{E}\left[\mathbb{I}_{B} \mathbb{I}_{\{S<T\}} \int_{[0, a]} \mathrm{p}_{S+}(v) d A_{v}\right],
\end{aligned}
$$

где последнее равенство следует из определения меры Кокса $\check{\mathbb{Q}}^{\circ}$. Процесс $\left(\int_{[0, a]} \mathrm{p}_{t+}(v) d A_{v}: t \in[a, \infty]\right)$ является $\mathbb{F}$-опциональным в соответствии с леммой 3.2, по теореме о сечениях (см. [8, теорема 4.12]) заключаем, что два процесса $M^{a}$ и $\left(\int_{[0, a]} \mathrm{p}_{t+}(v) d A_{v}: t \in[a, \infty]\right)$ являются $\mathbb{Q}$ неразличимыми на $[a, T)$. В частности, отображение $t \rightarrow \int_{[0, a]} \mathrm{p}_{t+}(v) d A_{v}$ является càdlàg процессом на $[a, T)$. В силу непрерывности справа по переменной $a$ процессы $M_{t}^{a}$ и $\int_{[0, a]} \mathrm{p}_{t+}(v) d A_{v}$ при $0 \leqslant a \leqslant t<T$ также неразличимы как двух-параметрические процессы. Теорема 4.2 доказана.

\section{5. Некоторые непосредственные следствия.}

Теорема 4.3. Предположим, ито выполнены те же условия, ито и в теореме 4.2. Процесс $\left(\mathrm{p}_{u+}(\omega, u): u \in[0, T)\right)$ является $\mathbb{F}$-прогрессивно измеримьм на $[0, T)$. Прочесс $\left(\int_{[0, t]} \mathrm{p}_{v+}(v) d A_{v}, t \in[0, T)\right)$, является $\mathbb{F}$-опчиональной дуальной проекцией возрастающего прочесса $\mathbb{I}_{[\tau, \infty)}$ на $[0, T)$.

Д о к а $з$ а т е л ь с т в о. В силу непрерывности справа по $t \in \mathbb{R}_{+}$ процесса $p_{t+}$ для любого $(\omega, u) \in \Omega \times[0, \infty]$ можем записать

$$
\mathrm{p}_{u+}(\omega, u)=\lim _{N \uparrow \infty} \sum_{k=1}^{\left\lfloor 2^{N} T\right\rfloor} \mathrm{p}_{v_{k}^{N}+}(\omega, u) \mathbb{I}_{\left\{v_{k-1}^{N} \leqslant u<v_{k}^{N}\right\}}, \quad t \in[0, T),
$$

где $v_{k}=k / 2^{N}$. Заметим, что для любых $k \leqslant\left\lfloor 2^{N} T\right\rfloor, a \in \mathbb{R}$ и $t \in[0, T)$ множество

$$
\left\{(\omega, u) \in \Omega \times[0, t]: \mathrm{p}_{v_{k}^{N}+}(\omega, u) \mathbb{I}_{\left\{v_{k-1}^{N} \leqslant u<v_{k}^{N}\right\}} \leqslant a\right\}
$$


лежит в

$$
\begin{aligned}
\left\{v_{k-1}^{N} \leqslant \widehat{\tau}<v_{k}^{N}\right\} & \cap\{\widehat{\tau} \leqslant t\} \cap \check{\mathscr{G}}_{v_{k}^{N}}+\left\{v_{k-1}^{N} \leqslant \widehat{\tau}<v_{k}^{N}\right\}^{c} \\
& \cap\{\widehat{\tau} \leqslant t\} \cap\{\emptyset, \Omega \times[0, \infty]\} \subset \mathscr{F}_{t+2 / 2^{N}} \otimes \mathscr{B}[0, t] .
\end{aligned}
$$

Следовательно, для любых $a \in \mathbb{R}$ и $t \in[0, T)$

$$
\left\{(\omega, u) \in \Omega \times[0, t]: \mathrm{p}_{u+}(\omega, u) \leqslant a\right\} \in \mathscr{F}_{t+\varepsilon} \otimes \mathscr{B}[0, t]
$$

для любого $\varepsilon>0$. Применяя теперь [7, глава IV, $\left.{ }^{\circ} 14\right]$, получаем, что процесс $\left(\mathrm{p}_{u+}(\omega, u): u \in[0, T)\right)$ является $\mathbb{F}$-прогрессивно измеримым на $[0, T)$.

Пусть $K$ - ограниченный $\mathbb{F}$-опциональный процесс. Тогда процесс $\check{K}_{t}(\omega, u)=K_{t}(\pi(\omega, u)), t \in \mathbb{R}_{+},(\omega, u) \in \Omega \times[0, \infty]$, является $\check{\mathbb{F}}-$ опциональным (что может быть доказано обычным путем с использованием теоремы о монотонных классах). Применяя теорему 4.2 для $a \in[0, T)$, получаем

$$
\begin{aligned}
\mathbb{E}\left[K_{\tau} \mathbb{I}_{\{\tau \leqslant a\}}\right] & =\mathbb{E}\left[K_{\tau \wedge a} \mathbb{I}_{\{\tau \leqslant \tau \wedge a\}}\right]=\check{\mathbb{E}}\left[\check{K}_{\hat{\tau} \wedge a} \mathbb{I}_{\{\hat{\tau} \leqslant \hat{\tau} \wedge a\}}\right] \\
& =\check{\mathbb{E}}^{\circ}\left[\check{K}_{\hat{\tau} \wedge a} \mathbb{I}_{\{\hat{\tau} \leqslant \hat{\tau} \wedge a\}} \mathbf{p}_{(\hat{\tau} \wedge a)+}\right]=\check{\mathbb{E}}^{\circ}\left[\check{K}_{\hat{\tau}} \mathbb{I}_{\{\hat{\tau} \leqslant a\}} \mathbf{p}_{\hat{\tau}+}\right] \\
& =\mathbb{E}\left[\int_{[0, a]} K_{v} \mathbf{p}_{v+}(v) d A_{v}\right] .
\end{aligned}
$$

Процесс $\left(\int_{[0, t]} \mathbf{p}_{v+}(v) d A_{v}, t \in[0, T)\right)$, является $\mathbb{F}$-согласованным процессом ограниченной вариации. Теорема 4.3 доказана.

Следствие 4.1. Пусть выполнень условия теоремь 4.2. Для любого $b \in[0, T)$ и любой ограниченной $\check{\mathscr{G}}_{b}^{0}$-измеримой функции $h$ прочесс $\left(\int_{[0, b]} h(u) \mathrm{p}_{t+}(u) d A_{u}, t \in[b, T)\right)$ является càdlàg версией $(\mathbb{Q}, \mathbb{F})$ равномерно интегрируемого мартингала $\mathbb{E}\left[h(\tau) \mathbb{I}_{\{\tau \leqslant b\}} \mid \mathscr{F}_{t}\right], t \in[b, T)$.

3 а м е ч а н и е 4.4. Последнее следствие сильнее, чем лемма 4.1.

Д о к а з а т е л с с т в о. Для любого $\mathbb{F}$-момента остановки $S \geqslant b$, в соответствии с теоремой 4.2, можем записать

$$
\begin{aligned}
\mathbb{E}\left[\mathbb{I}_{\{S<T\}} \mathbb{E}\left[h(\tau) \mathbb{I}_{\{\tau \leqslant b\}} \mid \mathscr{F}_{S}\right]\right] & =\mathbb{E}\left[h(\tau) \mathbb{I}_{\{S<T\}} \mathbb{I}_{\{\tau \leqslant b\}}\right]=\check{\mathbb{E}}\left[h \mathbb{I}_{\{\check{S}<T\}} \mathbb{I}_{\{\hat{\tau} \leqslant b\}}\right] \\
& =\check{\mathbb{E}}^{0}\left[h \mathbb{I}_{\{\check{S}<T\}} \mathbb{I}_{\{\hat{\tau} \leqslant b\}} \mathbf{p}_{\check{S}+}\right] \\
& =\mathbb{E}\left[\mathbb{I}_{\{S<T\}} \int_{[0, b]} h(u) \mathbf{p}_{S+}(u) d A_{u}\right] .
\end{aligned}
$$

Заметим, что процесс $\mathbb{I}_{\{\hat{\tau} \leqslant b\}} h \mathbb{1}_{[b, T)} \mathrm{p}_{+}$- $\check{\mathbb{G}}^{0}$-опциональный. В соответствии с леммой 3.2 процесс $\left(\int_{[0, b]} h(u) \mathrm{p}_{t+}(u) d A_{u}, t \in[b, T)\right)-\mathbb{F}$-опциональный. Этот процесс является càdlàg $(\mathbb{Q}, \mathbb{F})$-равномерно интегрируемым мартингалом на $[b, T)$ и

$$
\int_{[0, b]} h(u) \mathrm{p}_{t+}(u) d A_{u}=\mathbb{E}\left[h(\tau) \mathbb{1}_{\{\tau \leqslant b\}} \mid \mathscr{F}_{t}\right] .
$$

Следствие 4.1 доказано. 
Следствие 4.2. Пусть вьполнень предположения теоремь 4.2 и $b \in[0, T)$. Тогда

$$
\int_{[0, b]} \mathbb{I}_{\left\{\mathrm{p}_{b+}(u)=0\right\}} \mathrm{p}_{t+}(u) d A_{u}=0
$$

для любого $t \in(b, T)$.

Д о к а з а т е л ь с т о. Заметим, что согласно следствию 4.1 $\int_{[0, b]} \mathbb{I}_{\left\{\mathrm{p}_{b+}(u)=0\right\}} \mathrm{p}_{t+}(u) d A_{u}$ есть càdlàg неотрицательный $(\mathbb{Q}, \mathbb{F})$-мартингал на $[0, T)$ и

$$
\int_{[0, b]} \mathbb{I}_{\left\{\mathrm{p}_{b+}(u)=0\right\}} \mathrm{p}_{t+}(u) d A_{u}=\mathbb{E}\left[\mathbb{I}_{\left\{\mathrm{p}_{b+}(\tau)=0\right\}} \mathbb{I}_{\{\tau \leqslant b\}} \mid \mathscr{F}_{t}\right]
$$

Взяв математическое ожидание, получаем

$$
\mathbb{E}\left[\mathbb{I}_{\left\{\mathrm{p}_{b+}(\tau)=0\right\}} \mathbb{I}_{\{\tau \leqslant b\}}\right]=\check{\mathbb{E}}\left[\mathbb{I}_{\left\{\mathrm{p}_{b+}=0\right\}} \mathbb{I}_{\{\hat{\tau} \leqslant b\}}\right]=\check{\mathbb{E}}^{0}\left[\mathbb{I}_{\left\{\mathrm{p}_{b+}=0\right\}} \mathbb{I}_{\{\hat{\tau} \leqslant b\}} \mathrm{p}_{b+}\right]=0
$$

Следствие 4.2 доказано.

Далее приведем последнее следствие.

Следствие 4.3. Пусть $\left(M^{u}: u \in[0, \infty]\right)-\mathrm{i} M$-семейство, ассоииированное с $(\mathbb{Q}, \mathbb{F}, \tau)$. Пусть $A-\mathbb{F}$-согласованный неотрицательньй неубъвающий процесс, такой, ито $A_{\infty}=1$ и $A_{t}<1, t \in \mathbb{R}_{+}$. Пусть $\check{\mathbb{Q}}^{\circ}$ мера Кокса на вспомогательном произведении пространств, базирующаяся на $\check{A}$, ассоциированная $c(\check{\mathbb{Q}}, \check{\mathbb{F}}, \widehat{\tau})$. Тогда $\left(M^{u}: u \in[0, \infty]\right)$ дифференцируемо на $[0, T)$ относительно $A$ тогда и только тогда, когда $\check{\mathbb{Q}}$ абсолютно непрерьвна относительно $\check{\mathbb{Q}}^{\circ}$ на $\check{\mathscr{G}}_{t}^{0} \partial л я$ всех $t \in[0, T)$.

Д о к а з а т е л ь с т в о. Необходимость следует из теоремы 4.2. Докажем достаточность. Для $t \in[0, T)$ пусть $\xi$ обозначает функцию плотности $d \check{Q} / d \check{\mathbb{Q}}^{\circ}$ на $\check{\mathscr{G}}_{t}^{0}$. Существует $\mathscr{F}_{t} \otimes \mathscr{B}[0, \infty]$-измеримая функция $\mathrm{p}(\omega, u)$, такая, что

$$
\mathrm{p}(\widehat{\tau} \nmid t)=\check{\mathbb{E}}^{\circ}\left[\xi \mid \check{\mathscr{F}}_{t} \vee \sigma(\widehat{\tau} \nmid t)\right]
$$

Тогда для $B \in \mathscr{F}_{t}$ и $0 \leqslant u \leqslant t$

$$
\begin{aligned}
\mathbb{E}\left[\mathbb{I}_{B} M_{t}^{u}\right] & =\mathbb{E}\left[\mathbb{I}_{B} \mathbb{I}_{\{\tau \leqslant u\}}\right]=\check{\mathbb{E}}\left[\mathbb{I}_{B}(\pi) \mathbb{I}_{\{\hat{\tau} \leqslant u\}}\right] \\
& =\check{\mathbb{E}}^{\circ}\left[\mathbb{I}_{B}(\pi) \mathbb{I}_{\{\hat{\tau} \leqslant u\}} \xi\right]=\check{\mathbb{E}}^{\circ}\left[\mathbb{I}_{B}(\pi) \mathbb{I}_{\{\hat{\tau} \leqslant u\}} \mathrm{p}(\widehat{\tau} \nmid t)\right] \\
& =\mathbb{E}\left[\mathbb{I}_{B} \int_{[0, u]} \mathrm{p}(u) d A_{u}\right],
\end{aligned}
$$

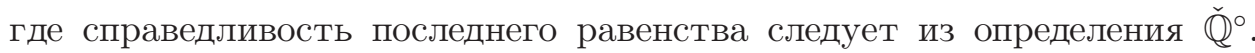
Следствие 4.3 доказано. 


\section{5. Модель, задаваемая стохастическим дифференциаль-} ным уравнением. В соответствии с теоремой 4.2 (следствие 4.3) для того, чтобы получить модель дифференцируемого момента, достаточно взять вероятностную меру, непрерывную относительно меры Кокса. Тем не менее, этот простой случай подходит не для всех практических ситуаций. Часто модель дефолта определяется другими специфическими свойствами, и в итоге для вычислительных целей дифференцируемость нужно доказывать. Например, модель динамической гауссовской копулы в [3], [25] определяется ее гауссовским свойством, и, как оказалось в дальнейшем, это соответствует модели начального момента.

В этом разделе приводится класс случайных моментов, введенный в [23] для динамического уравнения их і $M$-семейства. Эти моменты оказываются дифференцируемыми.

5.1. Возрастающее семейство мартингалов, выходящих из $1-Z$. Пусть $Z-(\mathbb{Q}, \mathbb{F})$-супермартингал, такой, что $0 \leqslant Z \leqslant 1$. Мы называем $Z$ супермартингалом Азема. Заметим, что для любого і $M$, процесс $\left(M_{u}^{u}\right)_{u \in \mathbb{R}_{+}}$является супермартингалом Азема. С другой стороны, в моделях рынков данные, калиброванные в соответствии с реальным рынком, могут быть представлены как супермартингал Азема $Z$. Важный вопрос состоит, следовательно, в том, существует ли модель, такая, что $\left(M_{u}^{u}\right)_{u \in \mathbb{R}_{+}}$совпадает с $Z$. Введем следующее определение (см. [12]).

О п р е д е л е н и е 5.1. і $M$-семейство $\left(M^{u}: u \in[0, \infty]\right)$ называется выходящим из $1-Z$, если семейство і $M$ удовлетворяет следующим условиям: для любых $0 \leqslant u \leqslant t<\infty, M_{u}^{u}=1-Z_{u}$ и $M_{t}^{u} \leqslant 1-Z_{t}$. Обозначим это семейство $M_{Z}(\mathbb{P}, \mathbb{F})$ или просто і $M_{Z}$.

Следующая теорема является непосредственным следствием теоремы 4.1 .

Теорема 5.1. Пусть $\left(M^{u}: u \in[0, \infty]\right)$ - iM-семейство, ассоииированное с $(\mathbb{Q}, \mathbb{F}, \tau)$. Пусть $Z$ - супермартингал Азема. Тогда $\left(M^{u}: u \in[0, \infty]\right)$ является і $M_{Z}$-семейством тогда и только тогда, когда $\mathbb{Q}\left[t<\tau \mid \mathscr{F}_{t}\right]=Z_{t}$ npu $t \geqslant 0$.

5.2. ६-уравнение и ६-пара. Зафиксируем супермартингал Азема $Z$. Пусть выполнено условие

Ну $(Z): 1-Z_{t}<0,1-Z_{t-}<0$ при $t \in(0, \infty)$.

Пусть $Z=M-A-$ это $(\mathbb{Q}, \mathbb{F})$-каноническое разложение $Z$ на $(\mathbb{Q}, \mathbb{F})$-локальный мартингал $M$ и неубывающий $\mathbb{F}$-предсказуемый процесс $A$, равный нулю в нулевой момент. Заметим, что ${ }^{\mathbb{F}} \cdot p(1-Z)_{t}=$ $1-Z_{t-}+\Delta_{t} A>0$ для любого $0<t<\infty$, где верхний индекс $\mathbb{F} \cdot p$ обозначает $(\mathbb{Q}, \mathbb{F})$-предсказуемую проекцию. Для $0<u<\infty$ определим $\widetilde{m}_{t}^{u}=\int_{u}^{t}-d M_{s} / \mathbb{F} \cdot p(1-Z)_{s}$ при $t \geqslant u$. Так как, очевидно, $d \widetilde{m}_{t}^{u}=d \widetilde{m}_{t}^{v}$ для 
$0<u<v \leqslant t<\infty$, то верхний индекс можно опустить и писать просто

$$
d \widetilde{m}_{t}=\frac{-d M_{t}}{\mathbb{F} \cdot p(1-Z)_{t}}, \quad t \in(0, \infty) .
$$

Пусть $\mathbb{D}$ - пространство всех càdlàg $\mathbb{F}$-адаптированных процессов. Пусть $m$ - целое положительное число и $\mathbf{Y}=\left(Y_{1}, \ldots, Y_{m}\right)-m$-мерный $(\mathbb{P}, \mathbb{F})$-локальный мартингал, $\mathbf{F}=\left(F_{1}, \ldots, F_{m}\right)$ - функционал Липшица из $\mathbb{D}$ в множество $m$-мерных локально ограниченных $\mathbb{F}$-предсказуемых процессов в смысле [18]. При $0<u<\infty$ для любой $\mathscr{F}_{u}$-измеримой случайной величины $x$ рассмотрим стохастическое дифференциальное уравнение, определяемое парой $(\mathbf{F}, \mathbf{Y})$ :

$$
\left(\natural_{u}\right)\left\{\begin{array}{l}
d X_{t}=X_{t-} d \tilde{m}_{t}+\mathbf{F}(X)_{t}^{\top} d \mathbf{Y}_{t}, \quad t \in[u, \infty), \\
X_{u}=x .
\end{array}\right.
$$

Будем называть пару $(\mathbf{F}, \mathbf{Y})$ Ł-парой, если она удовлетворяет следующему условию для любых $1 \leqslant j \leqslant m, u>0$ и любых $X, X^{\prime} \in \mathbb{D}$ :

(i) процесс

$$
t \in[u, \infty) \rightarrow \frac{F_{j}(X)_{t}}{\mathbb{F} \cdot p(1-Z)_{t}-X_{t-}} \mathbb{I}_{\left\{\left(1-Z_{t-}\right)-X_{t-} \neq 0\right\}}
$$

интегрируем относительно $Y_{j}$ и удовлетворяет неравенству $\Delta_{t} \tilde{m}-\frac{1}{\mathbb{F} \cdot p(1-Z)_{t}-X_{t-}} \mathbb{I}_{\left\{\mathbb{F} \cdot p(1-Z)_{t}-X_{t-} \neq 0\right\}} \mathbf{F}(X)_{t}^{\top} \Delta_{t} \mathbf{Y}>-1, \quad t \in[u, \infty)$

(ii) процесс

$$
t \in[u, \infty) \rightarrow \frac{F_{j}(X)_{t}}{X_{t-}} \mathbb{I}_{\left\{X_{t-} \neq 0\right\}}
$$

интегрируем относительно $Y_{j}$ и удовлетворяет неравенству

$$
\Delta_{t} \tilde{m}+\frac{1}{X_{t-}} \mathbb{I}_{\left\{X_{t-} \neq 0\right\}} \mathbf{F}(X)_{t}^{\top} \Delta_{t} \mathbf{Y} \geqslant-1, \quad t \in[u, \infty)
$$

(iii) если $0 \leqslant X, X^{\prime} \leqslant 1$, то процесс

$$
t \in[u, \infty) \rightarrow \frac{F_{j}(X)_{t}-F_{j}\left(X^{\prime}\right)_{t}}{X_{t-}-X_{t-}^{\prime}} \mathbb{I}_{\left\{X_{t-}-X_{t-}^{\prime} \neq 0\right\}}
$$

интегрируем относительно $Y_{j}$ и удовлетворяет неравенству

$\Delta_{t} \tilde{m}+\frac{1}{X_{t-}-X_{t-}^{\prime}} \mathbb{I}_{\left\{X_{t-}-X_{t-}^{\prime} \neq 0\right\}}\left(\mathbf{F}(X)_{t}-\mathbf{F}\left(X^{\prime}\right)_{t}\right)^{\top} \Delta_{t} \mathbf{Y} \geqslant-1, \quad t \in[u, \infty)$.

Следующая теорема (см. [23]) доказывает, что множество দ-пар непусто. 
Теорема 5.2. Пусть $g(t, x)$ - произвольная непрерьвно дифференцируемая функиия, определенная на $\mathbb{R}_{+} \times \mathbb{R}$, принимающая значения в $\mathbb{R}^{m}$. Пусть $\varphi \in C^{\infty}$ — возрастающая функиия на $\mathbb{R}_{+}$, такая, что $|\varphi(x)| \leqslant 2 u|\varphi(x) / x| \leqslant 1$. Введем множество $\mathrm{G}_{t}, t \in \mathbb{R}_{+}$, состоящее из элементов $\mathbf{z} \in \mathbb{R}^{m}$, удовлетворяющих двум условиям:

(a) $2\left|g(t, x)^{\top} \mathbf{z}\right|<1+\Delta_{t} \tilde{m} \quad$ nри $x \in \mathbb{R}$,

$$
\begin{aligned}
& {\left[-\varphi^{\prime}\left({ }^{\mathbb{F} \cdot p}\left(1-Z_{t-}\right)-x\right) \varphi(x) g(t, x)+\varphi\left({ }^{\mathbb{F} \cdot p}\left(1-Z_{t-}\right)-x\right) \varphi^{\prime}(x) g(t, x)\right.} \\
& \left.\quad+\varphi\left({ }^{\mathbb{F} \cdot p}\left(1-Z_{t-}\right)-x\right) \varphi(x) g^{\prime}(t, x)\right]^{\top} \mathbf{z}>-\left(1+\Delta_{t} \widetilde{m}\right) \quad \text { nрu } x \in \mathbb{R} .
\end{aligned}
$$

(Здесь $g^{\prime}(t, x)$ обозначает производную функиии $g(t, x)$ по $x$.) Тогда для любого $t \in \mathbb{R}_{+}$случайное множество $\mathrm{G}_{t}$ непусто, и множественнозначньй прочесс $\mathrm{G}$ является $\mathbb{F}$-опииональньл. Существует т-мерньии $\mathbb{F}$-локальный мартингал $\mathbf{Y}=\left(Y_{1}, \ldots, Y_{m}\right)$, скачок которого в момент $t \in \mathbb{R}_{+}$, если он происходит, содержится в $\mathrm{G}_{t}$. Пусть

$$
\mathbf{F}(X)_{t}=f\left(t, X_{t-}\right)=\varphi\left({ }^{\mathbb{F} \cdot p}(1-Z)_{t}-X_{t-}\right) \varphi\left(X_{t-}\right) g\left(t, X_{t-}\right), \quad X \in \mathbb{D} .
$$

Тогда сбормулированнье в начале разд. 5.2 условия (i), (ii) и (iii) при замене неравенства $\geqslant-1$ на строгое $>-1$ вылолнень для пары $(\mathbf{F}, \mathbf{Y})$.

5.3. Модель, задаваемая দ-уравнением. Более существенный вопрос, чем тот, что решается в теореме 5.1 , состоит в построении і $M_{Z}$ для заданного супермартингала Азема. দ-уравнение дает ответ на этот вопрос.

Теорема 5.3 (см. [12]). Пусть (F, Y) - некоторая দ-пара. Для $0<u<\infty$ рассмотрим уравнение $\left(\natural_{u}\right)$, соответствующее паре $(\mathbf{F}, \mathbf{Y})$. Пусть $\left(L_{t}^{u}: t \in[u, \infty)\right)$ - решение уравнения $\left(\bigsqcup_{u}\right)$ с начальным условием $L_{u}^{u}=1-Z_{u}$. Положим $L_{\infty}^{u}=\lim _{t \rightarrow \infty} L_{t}^{u}$,

$$
\begin{aligned}
& M_{u}^{u}=\left(1-Z_{u}\right), \\
& M_{t}^{u}=\inf _{v \in \mathrm{Q}, u<v \leqslant t}\left(L_{t}^{v}\right)^{+} \wedge\left(1-Z_{t}\right), \quad t \in(u, \infty],
\end{aligned}
$$

а также

$$
\begin{aligned}
M_{t}^{0} & =\inf _{u \in \mathbb{Q}, 0<u \leqslant t} M_{t}^{u}, \quad t \in(0, \infty], \\
M_{0}^{0} & =\lim _{t \downarrow 0} M_{t}^{0} \quad(\text { если существует }), \\
M_{t}^{\infty} & =1 \quad \text { для } t \in[0, \infty] .
\end{aligned}
$$

Тогда $M^{u} \mathbb{P}$-неразличимо с $L^{u}$ на $[u, \infty], 0<u<\infty, u\left(M^{u}: 0 \leqslant u \leqslant \infty\right)$ является ім ${ }_{Z}$-семейством.

Указанное выше і $M_{Z}$-семейство будем называть ассоциированным с দ-уравнением, также как и вероятностную меру $\mathbb{Q}^{\natural}$, построенную в теореме 4.1 для этого $\mathrm{i} M_{Z}$, будем называть ассоциированным с দуравнением. 
5.4. Дифференцируемость. Пусть $g \in C^{\infty}-$ некоторая функция на $\mathbb{R}$ с компактным носителем. Рассмотрим Ł-уравнение, ассоциированное со следующей типа:

$$
\begin{gathered}
\Delta_{t} Y \in \mathrm{G}_{t}, \\
\mathbf{F}(X)_{t}=\varphi\left({ }^{\mathbb{F} \cdot p}(1-Z)_{t}-X_{t-}\right) \varphi\left(X_{t-}\right) g\left(X_{t-}\right), \quad X \in \mathbb{D} .
\end{gathered}
$$

Предположим также, что $\varphi(x)=x$ при $x \in[0,1]$. Рассмотрим $\mathrm{i}_{Z}=$ $\left(M^{u}: 0 \leqslant u \leqslant \infty\right)$, ассоциированное с $(\mathbf{F}, \mathbf{Y})$. Так как $0 \leqslant M^{u} \leqslant 1-Z$, то

$$
\varphi\left({ }^{\mathbb{F} \cdot p}(1-Z)-M_{-}^{u}\right) \varphi\left(M_{-}^{u}\right)=\left({ }^{\mathbb{F} \cdot p}(1-Z)-M_{-}^{u}\right) M_{-}^{u} .
$$

Это означает, что $M^{u}$ удовлетворяет следующему стохастическому дифференциальному уравнению:

$$
\left\{\begin{array}{l}
d X_{t}=X_{t-} d \widetilde{m}_{t}+\left(\mathbb{F} \cdot p(1-Z)_{t}-X_{t-}\right) X_{t-} g\left(X_{t-}\right)^{\top} d \mathbf{Y}_{t}, \quad u \leqslant t<\infty \\
X_{u}=x
\end{array}\right.
$$

где $M_{u}^{u}=1-Z_{u}$. Теоремы 39 и 65 из [18, гл. V, разд. 10] применимы к такому уравнению. Пусть $x \rightarrow \Xi_{t}^{u}(x)$ - ассоциированный стохастический дифференциальный поток.

Теорема 5.4 (см. [23]). Пусть $0<t<\infty$,

$$
\kappa_{v}=\left(1+\Delta_{v} \widetilde{m}-\left(1-Z_{v-}\right) g\left(1-Z_{v-}\right)^{\top} \Delta_{v} \mathbf{Y}\right) \quad \text { nрu } v \in(0, t] .
$$

Тогда, если $\Delta_{v} A>0$, mо

$$
M_{t}^{v}-M_{t}^{v-}=\Xi_{t}^{v}\left(1-Z_{v}\right)-\Xi_{t}^{v}\left(1-Z_{v}-\kappa_{v} \Delta_{v} A\right) \quad \text { npu } v \in(0, t] .
$$

Eсли $\Delta_{v} A=0$, mo

$$
\begin{aligned}
& \lim _{u \uparrow v} \frac{M_{t}^{v}-M_{t}^{u}}{A_{v}-A_{u}}=\frac{d \Xi_{t}^{v}}{d x}\left(1-Z_{v}\right) \kappa_{v} \quad \text { npu } v \in(0, t], \\
& \lim _{u \downarrow v} \frac{M_{t}^{v}-M_{t}^{u}}{A_{v}-A_{u}}=\frac{d \Xi_{t}^{v}}{d x}\left(1-Z_{v}\right) \quad \text { npu } v \in(0, t) .
\end{aligned}
$$

Следовательно, $\mathrm{i} M_{Z}$ дифферениируемо относительно $A$ на $[0, \infty)$ c функиией плотности

$$
\begin{aligned}
& \mathrm{p}_{t}(u)=\frac{d \Xi_{t}^{u}}{d x}\left(1-Z_{u}\right) \mathbb{I}_{\left\{\Delta_{u} A=0\right\}} \\
& +\frac{\Xi_{t}^{u}\left(1-Z_{u}\right)-\Xi_{t}^{u}\left(1-Z_{u}-\kappa_{u} \Delta_{u} A\right)}{\Delta_{u} A} \mathbb{I}_{\left\{\Delta_{u} A>0\right\}}, \quad u \in(0, t], \quad t \in \mathbb{R}_{+} .
\end{aligned}
$$




\section{6. Копулы и порядковые статистики случайных моментов.}

В этом разделе мы изучаем свойство замкнутости при упорядочении класса дифференциальных моментов.

6.1. Упорядочение функций на $\{1, \ldots, k\}$. Напомним сначала о понятии порядковых статистик. Пусть $\mathfrak{a}$ - некоторая функция, определенная на $\{1, \ldots, k\}$ (где $k$ - положительное целое число), принимающая значения в $[0, \infty)$. Пусть $\left\{a_{1}, \ldots, a_{k}\right\}$ - множество значений $\mathfrak{a}$ и

$$
R^{\mathfrak{a}}(i)=R^{\left\{a_{1}, \ldots, a_{k}\right\}}(i)=\sum_{j=1}^{k} \mathbb{I}_{\left\{a_{j}<a_{i}\right\}}+\sum_{j=1}^{k} \mathbb{I}_{\left\{j<i, a_{j}=a_{i}\right\}}+1
$$

Отображение $i \in\{1, \ldots, k\} \rightarrow R^{\mathfrak{a}}(i) \in\{1, \ldots, k\}$ является биекцией. Пусть $\rho^{\mathfrak{a}}$ - обратное к нему отображение. Определим $\uparrow \mathfrak{a}=\mathfrak{a}\left(\rho^{\mathfrak{a}}\right)$. Легко видеть, что $\uparrow \mathfrak{a}$ является неубывающей функцией $\{1, \ldots, k\}$, принимающей те же значения, что и $\mathfrak{a}$. Величина $\uparrow \mathfrak{a}(i)$ представляет $i$-е по величине значение среди $\left\{a_{1}, \ldots, a_{k}\right\}$.

Пусть $k \in \mathbb{N}^{*}$ и $\tau_{1}, \ldots, \tau_{k}$ суть $k$ случайных моментов. Рассмотрим функцию $\mathfrak{t}$ на $\{1, \ldots, k\}$, принимающую соответственно значения $\left\{\tau_{1}, \ldots, \tau_{k}\right\}$. Положим $\sigma_{i}=\uparrow \mathfrak{t}(i), 1 \leqslant i \leqslant k$. Заметим, что, если $\tau_{i}$ - моменты остановки относительно некоторой фильтрации, то $\sigma_{i}$ - моменты остановки относительно той же фильтрации, так как

$$
\left\{\sigma_{i} \leqslant t\right\}=\cup_{I \subset\{1, \ldots, k\}, \sharp I=i}\left\{\tau_{j} \leqslant t \forall j \in I\right\}, \quad t \geqslant 0 .
$$

Это же уравнение показывает, что существует борелевская функция $\mathfrak{s}_{i}$ на $[0, \infty]^{k}$, такая, что $\sigma_{i}=\mathfrak{s}_{i}\left(\tau_{1}, \ldots, \tau_{k}\right)$.

Лемма 6.1. Пусть $t \in[0, \infty], 1 \leqslant i \leqslant k$. Обозначим $\mathfrak{S}_{i}=\{I \subset$ $\{1, \ldots, k\}: \sharp I=i\}$ u $B_{I}=\left\{\tau_{j} \leqslant u \forall j \in I\right\}$, где $I \subset\{1, \ldots, k\}$. Тогда

$$
\mathbb{Q}\left[\sigma_{i} \leqslant u \mid \mathscr{F}_{t}\right]=\sum_{S \subset \mathfrak{S}_{i}: S \neq \emptyset}(-1)^{1+|S|} \mathbb{Q}\left[\cap_{I \in S} B_{I} \mid \mathscr{F}_{t}\right]
$$

Д о к а з а т е ль с т в о. Имеем

$$
\mathbb{Q}\left[\sigma_{i} \leqslant u \mid \mathscr{F}_{t}\right]=\mathbb{Q}\left[\cup_{I \subset\{1, \ldots, k\}: \sharp I=i}\left\{\tau_{j} \leqslant u \forall j \in I\right\} \mid \mathscr{F}_{t}\right]=\mathbb{Q}\left[\cup_{I \in \mathfrak{S}_{i}} B_{I} \mid \mathscr{F}_{t}\right]
$$

По формуле включения-исключения последний член приобретает вид

$$
\mathbb{Q}\left[\cup_{I \in \mathfrak{S}_{i}} B_{I} \mid \mathscr{F}_{t}\right]=\sum_{S \subset \mathfrak{S}_{i}: S \neq \emptyset}(-1)^{1+|S|} \mathbb{Q}\left[\cap_{I \in S} B_{I} \mid \mathscr{F}_{t}\right]
$$

Лемма 6.1 доказана. 
6.2. Непрерывно дифференцируемые копулы. О понятии копулы см. [17].

Теорема 6.1. Пусть $C\left(x_{1}, \ldots, x_{k}\right)$ - непрерывно дифференцируемьле копульь. Пусть $\mathbf{M}^{i}=\left(M^{i, u}: u \in[0, \infty]\right), 1 \leqslant i \leqslant k,-\mathrm{i} M-$ семейства, удовлетворяющее условию дифференцируемости с горизонтом $T \in[0, \infty)$ относительно того же неубывающего прочесса $A$. Пусть $\tau_{i}, 1 \leqslant i \leqslant k$, - семейство случайньх моментов, такое, что их условное распределение относительно $\mathscr{F}_{T}$ задается (многомерной) функиией распределения $\left(u_{1}, \ldots, u_{k}\right) \rightarrow C\left(M_{T}^{1, u_{1}}, \ldots, M_{T}^{k, u_{k}}\right)$ nри $0 \leqslant$ $u_{1}, \ldots, u_{k} \leqslant T$. Тогда порядковые статистики для $\left(\tau_{i}, 1 \leqslant i \leqslant k\right)$ являются дифферениируемьми моментами для каждого горизонта $t \in[0, T]$ относительно $A$.

Д о к а з а т е л ь с т в о. Для любого $J \subset\{1, \ldots, k\}$ с помощью $C_{J}$ обозначим $J$-маргинальные копулы $C$, которые также непрерывно дифференцируемы. Пусть $\mathrm{p}_{T}^{i}$ - функция плотности с горизонтом $T$ для $\mathbf{M}^{i}$. Тогда при $0 \leqslant u \leqslant t \leqslant T$

$$
\begin{aligned}
\mathbb{Q}\left[\tau_{j} \leqslant u, j \in J \mid \mathscr{F}_{t}\right] & =\mathbb{Q}\left[\mathbb{Q}\left[\tau_{j} \leqslant u, j \in J \mid \mathscr{F}_{T}\right] \mid \mathscr{F}_{t}\right] \\
& =\mathbb{Q}\left[C_{J}\left(M_{T}^{1, u}, \ldots, M_{T}^{k, u}\right) \mid \mathscr{F}_{t}\right] .
\end{aligned}
$$

Применим формулу Ито (см. [9, формула $(2.52)])$ к функции $C_{J}\left(M_{T}^{1, u}\right.$ $\left.\ldots, M_{T}^{k, u}\right)$ относительно переменной $u$

$$
\begin{aligned}
& C_{J}\left(M_{T}^{1, u}, \ldots, M_{T}^{k, u}\right)=C_{J}\left(M_{T}^{1,0}, \ldots, M_{T}^{k, 0}\right) \\
& \quad+\sum_{j \in J} \int_{0}^{u} \frac{\partial C_{j}}{\partial x_{j}}\left(M_{T}^{1, u-}, \ldots, M_{T}^{k, u-}\right) \mathrm{p}_{T}^{j}(s) d A_{s} \\
& \quad+\sum_{s \in(0, u]} \mathbb{I}_{\left\{\Delta_{s} A>0\right\}}\left(\frac{C_{J}\left(M_{T}^{1, s}, \ldots, M_{T}^{k, s}\right)-C_{J}\left(M_{T}^{1, s-}, \ldots, M_{T}^{k, s-}\right)}{\Delta_{s} A}\right. \\
& \left.\quad-\sum_{j \in J} \frac{\partial C_{j}}{\partial x_{j}}\left(M_{T}^{1, s-}, \ldots, M_{T}^{k, s-}\right) \mathrm{p}_{T}^{j}(s)\right) \Delta_{s} A \\
& =\sum_{j \in J} \int_{0}^{u} \frac{\partial C_{j}}{\partial x_{j}}\left(M_{T}^{1, u-}, \ldots, M_{T}^{k, u-}\right) \mathrm{p}_{T}^{j}(s) d A_{s}^{c} \\
& \quad+\sum_{s \in(0, u]} \mathbb{I}_{\left\{\Delta_{s} A>0\right\}}\left(\frac{C_{J}\left(M_{T}^{1, s}, \ldots, M_{T}^{k, s}\right)-C_{J}\left(M_{T}^{1, s-}, \ldots, M_{T}^{k, s-}\right)}{\Delta_{s} A}\right) \Delta_{s} A,
\end{aligned}
$$

где $A^{c}$ обозначает непрерывную составляющую процесса $A$. Другими словами, существует $\mathscr{F}_{T} \otimes \mathscr{B}[0, \infty]$-измеримая функция $\xi_{J}(\omega, u)$, такая, что

$$
C_{J}\left(M_{T}^{1, u}, \ldots, M_{T}^{k, u}\right)=\int_{[0, u]} \xi_{J}(s) d A_{s}^{c}+\sum_{s \in[0, u]} \xi_{J}(s) \Delta_{s} A=\int_{[0, u]} \xi_{J}(s) d A_{s} .
$$


Следовательно,

$$
\mathbb{Q}\left[\tau_{j} \leqslant u, j \in J \mid \mathscr{F}_{t}\right]=\mathbb{Q}\left[\int_{[0, u]} \xi_{J}(s) d A_{s} \mid \mathscr{F}_{t}\right]=\int_{[0, u]} \mathbb{Q}\left[\xi_{J}(s) \mid \mathscr{F}_{t}\right] d A_{s} .
$$

Используя теперь те же обозначения, что и в лемме 6.1 , получим

$$
\begin{aligned}
\mathbb{Q}\left[\sigma_{i} \leqslant u \mid \mathscr{F}_{t}\right] & =\mathbb{Q}\left[\cup_{I \in \mathfrak{S}_{i}} B_{I} \mid \mathscr{F}_{t}\right] \\
& =\sum_{S \subset \mathfrak{S}_{i}: S \neq \emptyset}(-1)^{1+|S|} \mathbb{Q}\left[\cap_{I \in S} B_{I} \mid \mathscr{F}_{t}\right] \\
& =\sum_{S \subset \mathfrak{S}_{i}: S \neq \emptyset}(-1)^{1+|S|} \mathbb{Q}\left[B_{\cup_{I \in S} I} \mid \mathscr{F}_{t}\right] \\
& =\sum_{S \subset \mathfrak{S}_{i}: S \neq \emptyset}(-1)^{1+|S|} \int_{[0, u]} \mathbb{Q}\left[\xi_{\cup_{I \in S} I}(s) \mid \mathscr{F}_{t}\right] d A_{s} \\
& =\int_{[0, u]}\left(\sum_{S \subset \mathfrak{S}_{i}: S \neq \emptyset}(-1)^{1+|S|} \mathbb{Q}\left[\xi_{\cup_{I \in S} I}(s) \mid \mathscr{F}_{t}\right]\right) d A_{s} .
\end{aligned}
$$

Теорема 6.1 доказана.

3 а м е ч а н и е 6.1. В теореме 6.1 сформулирован принцип инвариантности для класса дифференцируемых моментов. Существует другой очевидный принцип инвариантности, а именно, класс дифференцируемых моментов инвариантен относительно абсолютно непрерывной замены вероятностной меры.

7. Условное математическое ожидание. В этом разделе будем предполагать, что выполнены те же условия, что и в теореме 4.2. В частности, $Z$ обозначает супермартингал Азема от $\tau$, который предполагается дифференцируемым на интервале $[0, T)$ относительно $A$ с функцией плотности р. Этот раздел посвящен вычислению условного математического ожидания при этих условиях.

7.1. Параметризованная опциональная проекция. Напомним, что в $[24$, утверждение 3$]$ для любой неотрицательной $\mathscr{B}\left(\mathbb{R}_{+}\right) \otimes \mathscr{A} \otimes$ $\mathscr{B}[0, \infty]$-измеримой функции $H(t, \omega, u)$ строится неотрицательная $\mathscr{O}(\mathbb{F}) \otimes$ $\mathscr{B}[0, \infty]$-измеримая функция, обозначаемая ${ }^{\circ} H$, такая, что для любого $u \in[0, \infty]^{\circ} H(u)$ является версией $(\mathbb{Q}, \mathbb{F})$-опциональной проекции процесса $H(u)$. В дальнейшем мы введем некоторый аналог понятия, используемого в [24].

Пусть $\mathbf{C}$ - семейство ограниченных $\mathscr{F}_{\infty} \otimes \mathscr{B}[0, \infty]$-измеримых функций, таких, что для каждого элемента $F$ этого семейства существует $\mathscr{O}(\mathbb{F}) \otimes \mathscr{B}[0, \infty]$-измеримая функция, обозначаемая $\left({ }^{\circ} F\right)_{t}(\omega, u),(t, \omega, u) \in$ $\mathbb{R}_{+} \times \Omega \times[0, \infty]$, удовлетворяющая следующим условиям:

(i) процесс $\left({ }^{\circ} F\right)_{t}(t), t \in \mathbb{R}_{+}$, является $\mathbb{F}$-опциональным;

(ii) для любого $u \in[0, \infty],\left({ }^{\circ} F\right)_{t}(u), t \in \mathbb{R}_{+}$, есть версия $(\mathbb{Q}, \mathbb{F})$ опциональной проекции $F(u)$; 
(iii) для любого $\mathbb{F}$-момента остановки $U$ процесс $\mathbb{I}_{\{U \leqslant t\}}\left({ }^{\circ} F\right)_{t}(U), t \in$ $\mathbb{R}_{+}$, является $\mathbb{F}$-опциональным. Для любой пары $\mathbb{F}$-моментов остановки $U, S$, таких, что $U \leqslant S$, и для любого $B \in \mathscr{F}_{S}$

$$
\mathbb{E}\left[\mathbb{I}_{B} F(U)\right]=\mathbb{E}\left[\mathbb{I}_{B}\left({ }^{\circ} F\right)_{S}(U)\right] .
$$

Ясно, что семейство $\mathbf{C}$ является линейным пространством и содержит функцию $F \equiv 1$. Заметим, что, если $F$ и $F^{\prime}$ - два элемента семейства $\mathbf{C}$, таких, что $0 \leqslant F \leqslant F^{\prime}$, то по теореме о сечениях (см. [8]) процесс $\mathbb{I}_{\{U \leqslant t\}}\left({ }^{\circ} F\right)_{t}(U), t \in \mathbb{R}_{+}$, ограничен снизу нулем и оценивается сверху процессом $\mathbb{I}_{\{U \leqslant t\}}\left({ }^{\circ} F^{\prime}\right)_{t}(U), t \in \mathbb{R}_{+} \cdot$ Пусть $F$ - ограниченная неотрицательная $\mathscr{F}_{\infty} \otimes \mathscr{B}[0, \infty]$-измеримая функция, являющаяся пределом возрастающей последовательности $\left(F_{n}\right)_{n \in \mathbb{N}^{*}}$ в С. Для любого $n \in \mathbb{N}$ пусть ${ }^{\circ} F_{n}-\mathscr{O}(\mathbb{F}) \otimes \mathscr{B}[0, \infty]$-измеримая функция, удовлетворяющая приведенным выше условиям (i)-(iii) для $F_{n}$. Положим ${ }^{\circ} F=\sup _{n \in \mathbb{N}^{*}}{ }^{\circ} F_{n}$. Тогда ${ }^{\circ} F-\mathscr{O}(\mathbb{F}) \otimes \mathscr{B}[0, \infty]$-измеримая функция, и она удовлетворяет условиям (i)-(iii) для $F$. Это наблюдение показывает, что семейство C - функциональный монотонный класс в смысле [8, теорема 1.4]. Для любых $C \in \mathscr{F}_{\infty}$ и $D \in \mathscr{B}[0, \infty]$, полагая $\left({ }^{\circ} F\right)_{t}(u)=\mathbb{E}\left[\mathbb{I}_{C} \mid \mathscr{F}_{t}\right] \mathbb{I}_{D}(u)$, мы видим, что семейство $\mathbf{C}$ содержит все функции вида $F=\mathbb{I}_{C} \mathbb{I}_{D}$. По теореме о монотонных классах (см. [8, теорема 1.4]) семейство $\mathbf{C}$ содержит все ограниченные $\mathscr{F}_{\infty} \otimes \mathscr{B}[0, \infty]$-измеримые функции.

$\mathrm{C}$ помощью предельного перехода мы расширяем определение ${ }^{\circ} \mathrm{F}$ с семейства $\mathbf{C}$ на все неотрицательные функции $F$. Теперь мы можем сформулировать следующее определение.

О п р е д е л е н и е 7.1. Для любой ограниченной или неотрицательной $\mathscr{F}_{\infty} \otimes \mathscr{B}[0, \infty]$-измеримой функции $F$ обозначим ${ }^{\circ} F$ любую $\mathscr{O}(\mathbb{F}) \otimes \mathscr{B}[0, \infty]$-измеримую функцию, удовлетворяющую условиям (i)(iii).

3 а м е ч а н и е 7.1. Заметим, что, если измеримое пространство $(\Omega, \mathscr{A})$ имеет хорошее топологическое свойство, то функция ${ }^{\circ} \mathrm{F}$ может быть определена более точно с помощью процесс прогнозирования [1].

Следующая лемма является прямым следствием определения ${ }^{\circ} \mathrm{F}$.

Лемма 7.1. Пусть $F$ - любая ограниченная или неотрицательная $\mathscr{F}_{\infty} \otimes \mathscr{B}[0, \infty]$-измеримая функиия и $U-\mathbb{F}$-момент остановки. Если $F(U)$ интегрируема, то отображения ${ }^{\circ} F_{t}(U), t \in[U, \infty)$, определяют $(\mathbb{Q}, \mathbb{F})$-равномерно интегрируемьй са̀dlàg мартингал на $[U, \infty)$. Следовательно, $\mathbb{F}$-предсказуемая проекиия $F(U)$ на $[U, \infty)$ определяется $\operatorname{\kappa a\kappa }^{\circ} F_{t-}(U), t \in[U, \infty)$.

Лемма 7.2. Для любого $\mathbb{F}$-адаптированного неотрииательного неубивающего прочесса $A$, любой ограниченной или неотрицательной $\mathscr{F}_{\infty} \otimes \mathscr{B}[0, \infty]$-измеримой функиии $F$ и для любой версии ${ }^{\circ} F$ справедливо 
равенство

$$
\mathbb{E}\left[\int_{[0, t]} f(u) F(u) d A_{u}\right]=\mathbb{E}\left[\int_{[0, t]} f(u)^{\circ} F_{t}(u) d A_{u}\right]
$$

для любого $t \in \mathbb{R}_{+}$и любой ограниченной $\cap_{s>t}\left(\mathscr{F}_{s} \otimes \mathscr{B}[0, \infty]\right)$-измеримой функиии $f$.

Д о к а з а т е л ь с в о. Приведем доказательство для случая $F \geqslant 0$. Пусть $c(s)=\inf \left\{t \in \mathbb{R}_{+}: A_{t}>s\right\}$. Используя результаты раздела 2.4., получим

$$
\begin{gathered}
\mathbb{E}\left[\int_{[0, t]} f(u) F(u) d A_{u}\right]=\mathbb{E}\left[\int_{[0, \infty)} \mathbb{I}_{\{c(s) \leqslant t\}} f(c(s)) F(c(s)) d s\right] \\
\quad=\int_{[0, \infty)} \mathbb{E}\left[\mathbb{I}_{\{c(s) \leqslant t\}} f(c(s)) F(c(s))\right] d s \text { (по теореме Фубини) } \\
\quad=\int_{[0, \infty)} \mathbb{E}\left[\mathbb{I}_{\{c(s) \leqslant t\}} f(c(s))\left({ }^{\circ} F\right)_{t}(c(s))\right] d s
\end{gathered}
$$

(так как $c(s)-\mathbb{F}$-момент остановки

и $\mathbb{I}_{\{c(s) \leqslant t\}} f(c(s))$ является $\mathscr{F}_{t}$-измеримой)

$$
=\mathbb{E}\left[\int_{[0, t]} f(u)^{\circ} F_{t}(u) d A_{u}\right] .
$$

Лемма 7.2 доказана.

Лемма 7.3. Если выполнень условия леммь 7.2, то справедливо соотношение

$$
\check{\mathbb{E}}^{\circ}\left[F \mid \check{\mathscr{G}}_{t}^{0}\right] \mathbb{I}_{\{\hat{\tau} \leqslant t\}}={ }^{\circ} F_{t} \mathbb{I}_{\{\hat{\tau} \leqslant t\}} .
$$

Д о к а з а т е л ь с т в о. Перепишем выкладки предыдущей леммы в следующем виде:

$$
\begin{aligned}
\check{\mathbb{E}}^{\circ}\left[f \mathbb{I}_{\{\hat{\tau} \leqslant t\}} F\right] & =\mathbb{E}\left[\int_{[0, t]} f(u) F(u) d A_{u}\right] \\
& =\mathbb{E}\left[\int_{[0, t]} f(u)^{\circ} F_{t}(u) d A_{u}\right]=\check{\mathbb{E}}^{\circ}\left[f \mathbb{I}_{\{\hat{\tau} \leqslant t\}}{ }^{\circ} F_{t}\right] .
\end{aligned}
$$

Лемма 7.3 доказана.

7.2. Формулы условного математического ожидания. В этом подразделе мы покажем, что условное математическое ожидание относительно $\mathscr{G}_{t}$ может быть вычислено в терминах функции плотности р и условного распределения относительно $\mathscr{F}_{t}$.

Приведенная ниже лемма обычно в литературе называется ключевой (см. [4, лемма 3.1.2]). Напомним, что $Z_{t}=\mathbb{E}\left[\mathbb{I}_{\{t<\tau\}} \mid \mathscr{F}_{t}\right]$.

Лемма 7.4. Для любого ограниченного $\mathrm{H} \in \mathscr{G}_{\infty}$,

$$
\mathbb{E}\left[\mathrm{H} \mid \mathscr{G}_{t}\right] \mathbb{I}_{\{t<\tau\}}=\frac{\mathbb{E}\left[\mathrm{H} \mathbb{I}_{\{t<\tau\}} \mid \mathscr{F}_{t}\right]}{Z_{t}} \mathbb{I}_{\{t<\tau\}}, \quad t \in[0, \infty) .
$$


Д о к а з а т е л ь с т в о. Пусть сначала $h-$ ограниченная $\mathscr{F}_{t} \otimes$ $\mathscr{B}[0, \infty]$-измеримая функция. Тогда

$$
\begin{aligned}
\mathbb{E}\left[h(\tau \nmid t) \mathbb{1}_{\{t<\tau\}} \mathrm{H}\right] & =\mathbb{E}\left[h(\infty) \mathbb{I}_{\{t<\tau\}} \mathrm{H}\right]=\mathbb{E}\left[h(\infty) \mathbb{E}\left[\mathrm{H} \mathbb{1}_{\{t<\tau\}} \mid \mathscr{F}_{t}\right]\right] \\
& =\mathbb{E}\left[h(\infty) \mathbb{I}_{\{t<\tau\}} \frac{\mathbb{E}\left[\mathrm{H} \mathbb{I}_{\{t<\tau\}} \mid \mathscr{F}_{t}\right]}{\left.\mathbb{E}\left[\mathbb{1}_{\{t<\tau\}} \mid \mathscr{F}_{t}\right]\right]}\right. \\
& =\mathbb{E}\left[h(\tau \nmid t) \mathbb{I}_{\{t<\tau\}} \frac{\mathbb{E}\left[\mathrm{H} \mathbb{I}_{\{t<\tau\}} \mid \mathscr{F}_{t}\right]}{Z_{t}}\right] .
\end{aligned}
$$

Заметим, что все величины в этой формуле ограничены. Следовательно, можно рассмотреть правый предел по $t \in \mathbb{R}_{+}$и заключить, что то же самое соотношение остается справедливым, если $h(\tau \nmid t)$ заменить ограниченной $\mathscr{G}_{t}$-измеримой функцией. Лемма 7.4 доказана.

Напомним, что отображение $\phi$ было введено в разд. 3.2.

Теорема 7.1. Пусть выполнены предположения теоремь 4.2 u $b \in$ $[0, T)$. Для любой неотричательной $\check{\mathscr{G}}_{b}^{0}$-измеримой функции $f$ nри $t \in$ $[0, b]$

$$
\mathbb{E}\left[f(\phi) \mid \mathscr{G}_{t}^{0}\right]=\frac{\mathbb{E}\left[f(\phi) \mathbb{I}_{\{t<\tau\}} \mid \mathscr{F}_{t}\right]}{Z_{t}} \mathbb{I}_{\{t<\tau\}}+\frac{{ }^{\circ}\left(f \mathrm{p}_{b+}\right)_{t}(\tau)}{\mathrm{p}_{t+}(\tau)} \mathbb{1}_{\left\{\mathrm{p}_{t+}(\tau)>0\right\}} \mathbb{I}_{\{\tau \leqslant t\}}
$$

Д о к а з а т е л ь с т в о. На множестве $\{t<\tau\}$ данная формула является следствием леммы 7.4. Рассмотрим ее же на множестве $\{\tau \leqslant t\}$. Заметим, что

$$
\mathbb{E}\left[f(\phi) \mid \mathscr{G}_{t}^{0}\right] \mathbb{I}_{\{\tau \leqslant t\}}=\left(\check{\mathbb{E}}\left[f \mid \check{\mathscr{G}}_{t}^{0}\right] \mathbb{I}_{\{\hat{\tau} \leqslant t\}}\right)(\phi) .
$$

Применим теорему 4.2 .

$$
\begin{aligned}
\check{\mathbb{E}}\left[f \mid \check{\mathscr{G}}_{t}^{0}\right] \mathbb{I}_{\{\hat{\tau} \leqslant t\}} & =\frac{1}{\mathrm{P}_{t}} \check{\mathbb{E}}^{\circ}\left[f \mathbb{I}_{\{\hat{\tau} \leqslant b\}} \mathrm{P}_{b} \mid \check{\mathscr{G}}_{t}^{0}\right] \mathbb{I}_{\left\{\mathrm{P}_{t}>0\right\}} \mathbb{I}_{\{\hat{\tau} \leqslant t\}} \\
& =\frac{1}{\mathrm{p}_{t+}} \check{\mathbb{E}}^{\circ}\left[f \mathrm{p}_{b+} \mid \check{\mathscr{G}}_{t}\right] \mathbb{I}_{\left\{\mathrm{p}_{t+}>0\right\}} \mathbb{I}_{\{\hat{\tau} \leqslant t\}} \\
& =\frac{{ }^{\circ}\left(f \mathrm{p}_{b+}\right)_{t}}{\mathrm{p}_{t+}} \mathbb{I}_{\left\{\mathrm{p}_{t+}>0\right\}} \mathbb{I}_{\{\tau \leqslant t\}},
\end{aligned}
$$

в соответствии с леммой 7.3. Теорема 7.1 доказана.

Следствие 7.1. Пусть $b \in[0, T) u t \in[0, b]$. Имеем

$$
\mathbb{I}_{\{\tau \leqslant t\}} \mathrm{p}_{t+}(\tau)=\mathbb{1}_{\{\tau \leqslant t\}}{ }^{o}\left(\mathrm{p}_{b+}\right)_{t}(\tau) \quad u \quad \mathbb{1}_{\left\{\mathrm{p}_{t+}(\tau)>0\right\}} \mathbb{I}_{\{\tau \leqslant t\}}=\mathbb{1}_{\{\tau \leqslant t\}},
$$

$\mathbb{Q}$ почти наверное. Более того, ${ }^{\circ}\left(\mathrm{p}_{b+}\right)_{t}(\omega, u)$ - еще одна версия функчии плотности с горизонтом $t$. 
Д о к а з а т е л ь с т в о. Если положить $f \equiv 1$ в формуле в теореме 7.1, получим

$$
\mathbb{I}_{\{\tau \leqslant t\}}=\frac{\circ\left(\mathrm{p}_{b+}\right)_{t}(\tau)}{\mathrm{p}_{t+}(\tau)} \mathbb{I}_{\left\{\mathrm{p}_{t+}(\tau)>0\right\}} \mathbb{I}_{\{\tau \leqslant t\}},
$$

что доказывает первую часть леммы. Вторая часть является следствием тождества

$$
\mathbb{E}\left[h(\tau) \mathbb{I}_{\{\tau \leqslant t\}}\right]=\mathbb{E}\left[\int_{[0, t]} h(u)^{\circ}\left(\mathrm{p}_{b+}\right)_{t}(u) d A_{u}\right]
$$

для любой ограниченной $\check{\mathscr{G}}_{t}^{0}$-измеримой функции $h$, вследствие леммы 7.2.

3 а м е ч а н и е 7.2. Заметим, что новая версия ${ }^{\circ}\left(\mathrm{p}_{b+}\right)_{t}(u)$ функции плотности имеет более простую измеримость, чем $\mathrm{p}_{t+}$. Действительно, ${ }^{\circ}\left(\mathrm{p}_{b+}\right)_{t}(u)$ является $\mathscr{F}_{t} \otimes \mathscr{B}[0, \infty]$-измеримой при $t \in[0, b]$. Еще одно замечание связано с тем, что для каждого $u \in[0, \infty]$, если $\mathrm{p}_{b+}(u)$ является $\mathbb{Q}$-интегрируемой функцией, то $t \rightarrow{ }^{\circ}\left(\mathrm{p}_{b+}\right)_{t}(u)$ является $(\mathbb{Q}, \mathbb{F})$ равномерно интегрируемым мартингалом.

8. Формула опционального расщепления. Одно из важных свойств полезной модели риска дефолта — это формула опционального расщепления (см. [21]). Это формула связана с вопросом измеримости, необходимой в различных ситуациях для справедливости вычислений на основе условных распределений. См. более подробно в [21]. Заметим, что формула опционального расщепления справедлива для начальных моментов. В этом разделе мы докажем его для дифференцируемых

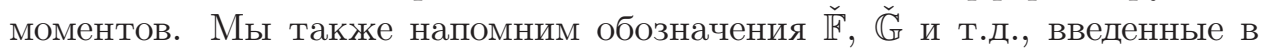
разд. 3.2 .

Теорема 8.1. Предположим, что выполнень предположения теоремь 4.2. Формула опционального расщепления на $[0, T)$ выполнена на $\oint$ фьтрации $\mathbb{G}$ для вероятностной мерь $\mathbb{Q}$. Это означает, ито для любого $\mathbb{G}$-опиионального процесса $X$ существует $\mathbb{F}$-опциональньии проиесс $X^{\prime}$ и $\mathscr{O}(\mathbb{F}) \otimes \mathscr{B}[0, \infty]$-измеримая функиия $X^{\prime \prime}$, такие, что выполнено следуюшее (с точностью до $\mathbb{Q}$-неразличимости) тождество:

$$
X \mathbb{I}_{[0, T)}=X^{\prime} \mathbb{I}_{[0, \tau)} \mathbb{I}_{[0, T)}+X^{\prime \prime}(\tau) \mathbb{I}_{[\tau, \infty)} \mathbb{I}_{[0, T)} .
$$

Д о к а з а т е ль с т в о. Пусть $X$ есть $\mathbb{G}$-опциональный процесс. Из [21] известно, что всегда существует $\mathbb{F}$-опциональный процесс $X^{\prime}$, такой, что $X \mathbb{1}_{[0, \tau)}=X^{\prime} \mathbb{1}_{[0, \tau)}$. Нужно доказать только формулу опционального расщепления на интервале $[\tau, \infty) \cap[0, T)$.

В соответствии с [8, теорема 4.36] существует $\mathbb{G}^{0}$-опциональный процесс $X^{0}, \mathbb{Q}$-неразличимый с $X$. По лемме 3.1 с учетом [8, теорема 1.5$]$ существует $\check{\mathbb{G}}^{0}$-опциональный процесс $\bar{X}$, такой, что $X^{0}=\bar{X}(\Phi)$. 
Пусть $\mathscr{Q}^{\circ}$ - мера Кокса на вспомогательном произведении пространств, базирующаяся на $\check{A}$, ассоциированная с $(\check{\mathbb{Q}}, \check{\mathbb{F}}, \widehat{\tau})$. Для меры Кокса $\check{Q}^{\circ}$ гипотеза $(H)$ (см. [6, лемма 3.2.1]) удовлетворяется, так что в соответствии с [21] формула опционального расщепления выполнена. Следовательно, выполнено (с точностью до $\check{\mathbb{Q}}^{\circ}$-неразличимости) тождество

$$
\bar{X}=\bar{X}^{\prime} \mathbb{I}_{[0, \hat{\tau})}+\bar{X}^{\prime \prime}(\widehat{\tau}) \mathbb{I}_{[\hat{\tau}, \infty)}
$$

для $\check{\mathbb{F}}$-опционального процесса $\bar{X}^{\prime}$ и $\mathscr{O}(\check{\mathbb{F}}) \otimes \mathscr{B}[0, \infty]$-измеримой функции

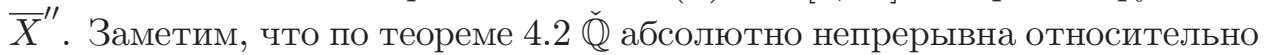
$\check{\mathbb{Q}}^{\circ}$ на $\check{\mathscr{G}}_{b}^{\circ}$ для любого $b \in[0, T)$. Следовательно,

$$
\bar{X} \mathbb{I}_{[\hat{\tau}, T)}=\bar{X}^{\prime \prime}(\widehat{\tau}) \mathbb{I}_{[\hat{\tau}, T)}
$$

— также тождество (с точностью до Ф্Q-неразличимости). Отображение $\bar{X}^{\prime \prime}$ является функцией четырех переменных:

$$
\bar{X}_{t}^{\prime \prime}((\omega, u), x), \quad(t,(\omega, u)) \in \mathbb{R}_{+} \times(\Omega \times[0, \infty]), \quad x \in[0, \infty] .
$$

Определим функцию $X_{t}^{\prime \prime}(\omega, x)=\bar{X}_{t}^{\prime \prime}(\phi(\omega), x)\left(X^{\prime \prime}(x)=\bar{X}^{\prime \prime}(\Phi, x)\right)$. С помощью теоремы о монотонных классах и с использованием рассуждений в доказательстве леммы 3.1, можно показать, что она является $\mathscr{O}(\mathbb{F}) \otimes \mathscr{B}[0, \infty]$-измеримой функций. Тогда получим следующее (с точностью до $\mathbb{Q}$-неразличимости) тождество:

$$
X^{0} \mathbb{1}_{[\tau, T)}=X^{\prime \prime}(\tau) \mathbb{1}_{[\tau, T)} .
$$

Теорема 8.1 доказана.

Следствие 8.1. Для любого $t \in[0, T) \sigma$-алгебра $\mathscr{F}_{t} \vee \sigma(\tau \nmid t)$, дополненная $\left(\mathbb{Q}, \mathscr{G}_{\infty}\right)$-нулевьли множествами, совпадает с $\mathscr{G}_{t}$. Более того, $\sigma\left(H_{\tau}: H-\mathbb{F}\right.$-опциональньй процесс), дополненная $\left(\mathbb{Q}, \mathscr{G}_{\infty}\right)$ нулевьли множествами, совпадает с $\mathscr{G}_{\tau}$.

Д о к а з а т е л с т в о. Утверждение является следствием предыдущей теоремы и теорем 3.4 и 3.6 из [21].

3 а м е ч а н и е 8.1. Свойство, доказанное в предыдущем следствии, важно с точки зрения результатов [4], где указанное свойство при условиях, обозначаемых там G.1 и G.2, требуется для установления этих результатов.

9. Формула расширения фильтрации. В этом разделе мы докажем, что прогрессивное расширение фильтрации с дифференцируемым моментом удовлетворяет гипотезе $\left(H^{\prime}\right)$, состоящей в том, что $\mathbb{F}$ мартингал $X$ является $\mathbb{G}$-семимартингалом. Мы найдем соответствующее семимартингальное разложение $X$ на $\mathbb{G}$. Предположим, что выполнены те же условия и замечания, что и в теореме 4.2. Напомним, что $Z$ 
обозначает супермартингал Азема от $\tau$ и $M$ обозначает его мартингальную составляющую (на $\mathbb{F}$ ). Начнем со следующего хорошо известного результата (см. [14], [15], [13]).

Лемма 9.1. Пусть $X$ - ограниченный $(\mathbb{Q}, \mathbb{F})$-мартингал и $B^{X}-$ $(\mathbb{Q}, \mathbb{F})$-предсказуемая дуальная проекиия скачкообразного прочесса $t \rightarrow$ $\Delta X_{\tau} \mathbb{I}_{\{0<\tau \leqslant t\}} \cdot$ Тогда

$$
X_{\cdot \wedge \tau}-\int_{0}^{\cdot \wedge \tau} \frac{1}{Z_{s-}}\left(d\langle M, X\rangle_{s}+d B_{s}^{X}\right)
$$

является $(\mathbb{Q}, \mathbb{G})$-локальньл мартингалом, где предсказуемая скобка $\langle\cdot, \cdot\rangle=\left\langle\cdot, \cdot{ }^{\mathbb{Q} \cdot \mathbb{F}}\right.$ вьчиислена для $(\mathbb{Q}, \mathbb{F})$.

Лемма 9.2. Для любого ограниченного $(\mathbb{Q}, \mathbb{F})$-мартингала $X$ прочесс $\check{X}=X(\pi)$ является $\left(\check{\mathbb{Q}}^{\circ}, \check{\mathbb{G}}\right)$-мартингалом. Существует $\mathscr{P}(\mathbb{F}) \otimes$ $\mathscr{B}[0, \infty]$-измеримая функиия $h_{t}(\omega, v),(t, \omega) \in \mathbb{R}_{+} \times \Omega, v \in[0, \infty]$, такая, чmo

$$
\mathbb{I}_{(\hat{\tau}, T)} \frac{d\langle\check{X}, \mathrm{P}\rangle^{\check{\mathbb{Q}}^{\circ} \cdot \check{G}}}{d\langle\check{X}, \check{X}\rangle \check{\mathbb{Q}}^{\circ} \cdot \check{G}}=\mathbb{I}_{(\hat{\tau}, T)} h(\pi, \widehat{\tau}) .
$$

Д о к а з а т е л с с в о. Первое утверждение верно в силу справедливости гипотезы $(H)$ для $\check{\mathbb{F}}$ в $\check{\mathbb{G}}$ с мерой Кокса $\check{\mathbb{Q}}^{\circ}$ (см. [6, разд. 3.2]). Что касается второго утверждения, заметим, что существует

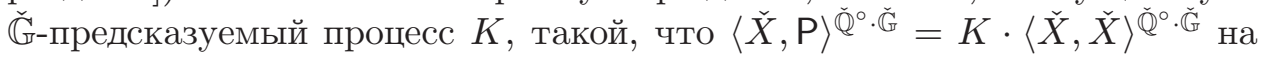
$[0, T)$. В соответствии с формулой предсказуемого расщепления (см. [13, лемма (4.4)]) существует $\mathscr{P}(\check{\mathbb{F}})$-измеримый процесс $K^{\prime}$ и $\mathscr{P}(\check{\mathbb{F}}) \otimes \mathscr{B}[0, \infty]-$ измеримая функция $K^{\prime \prime}(u)$, такие, что

$$
K=K^{\prime} \mathbb{I}_{[0, \hat{\tau}]}+K^{\prime \prime}(\widehat{\tau}) \mathbb{I}_{(\hat{\tau}, \infty)} .
$$

Далее, по теореме о монотонных классах, легко видеть, что $K^{\prime \prime}$ приобретает форму

$$
K_{t}^{\prime \prime}((\omega, u), v)=h_{t}(\pi(\omega, u), v)
$$

для некоторой $\mathscr{P}(\mathbb{F}) \otimes \mathscr{B}[0, \infty]$-измеримой функции $h_{t}(\omega, v),(t, \omega) \in \mathbb{R}_{+} \times$ $\Omega, v \in[0, \infty]$. Лемма 9.2 доказана.

Теорема 9.1. Для любого ограниченного $(\mathbb{Q}, \mathbb{F})$-мартингала $X$ проиесс

$$
X_{t}-X_{0}-\int_{0}^{t} \mathbb{I}_{\{s \leqslant \tau\}} \frac{1}{Z_{s-}}\left(d\langle M, X\rangle_{s}+d B_{s}^{X}\right)-\int_{0}^{t} \mathbb{I}_{\{\tau<s\}} \frac{h(\tau)}{\mathrm{p}_{s-}(\tau)} d\langle X, X\rangle_{s}
$$

является $(\mathbb{Q}, \mathbb{G})$-локальным мартингалом на интервале $t \in[0, T)$, где $\mathrm{p}_{-}$обозначает левьй предел для $s>0$ прочесса $\mathrm{p}_{+}$. 
Д о к а з а т е л ь с т в о. По лемме $9.1 X^{\tau}$ есть $(\mathbb{Q}, \mathbb{G})$-специальный семимартингал с процессом сноса

$$
\int_{0}^{t \wedge \tau} \frac{1}{Z_{s-}}\left(d\langle M, X\rangle_{s}+d B_{s}^{X}\right), \quad t \in \mathbb{R}_{+} .
$$

По теореме Гирсанова

$$
\check{X}-\check{X}^{\hat{\tau}}-\frac{1}{\mathrm{P}_{-}} \mathbb{1}_{(0, T)} \cdot\left\langle\check{X}-\check{X}^{\hat{\tau}}, \mathrm{P}\right\rangle^{\check{\mathbb{Q}}^{\circ} \cdot \check{G}}
$$

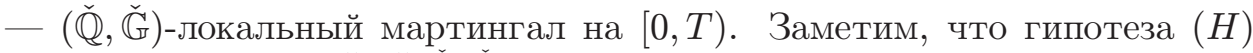
влечет равенство $\langle\check{X}, \check{X}\rangle^{\check{\mathbb{Q}}^{\circ} \cdot \check{G}}=\langle X, X\rangle(\pi)$. Следовательно,

$$
\begin{aligned}
& \frac{1}{\mathrm{P}_{-}} \mathbb{I}_{(0, T)} \cdot\left\langle\check{X}-\check{X}^{\hat{\tau}}, \mathrm{P}\right\rangle^{\mathscr{\mathbb { Q }}^{\circ} \cdot \check{G}}=\frac{1}{\mathrm{P}_{-}} \mathbb{I}_{(\hat{\tau}, T)} \cdot\langle\check{X}, \mathrm{P}\rangle \check{\mathbb{Q}}^{\circ} \cdot \check{G} \\
& \quad=\frac{K^{\prime \prime}(\widehat{\tau})}{\mathrm{p}_{-}} \mathbb{I}_{(\hat{\tau}, T)} \cdot\langle\check{X}, \check{X}\rangle^{\circ} \cdot \check{\mathbb{G}}^{\prime}=\frac{h(\pi, \widehat{\tau})}{\mathrm{p}_{-}} \mathbb{I}_{(\hat{\tau}, T)} \cdot\langle X, X\rangle(\pi) .
\end{aligned}
$$

Следует заметить, что для любого $\mathbb{G}$ локально ограниченный опциональный процесс $Y$ является $(\mathbb{Q}, \mathbb{G})$-локальным мартингалом на $[0, T]$ тогда и только тогда, когда $\check{Y}$ является локальным ( на $[0, T]$. Это позволяет применить мартингальное свойство к $\Omega$, чтобы заключить, что

$$
X-X^{\tau}-\frac{h(\tau)}{\mathrm{p}_{-}(\tau)}, \mathbb{I}_{(\tau, T)} \cdot\langle X, X\rangle
$$

является $(\mathbb{Q}, \mathbb{G})$-локальным мартингалом на $[0, T)$. Это завершает доказательство, так как $X=X^{\tau}+\left(X-X^{\tau}\right)$. Теорема 9.1 доказана.

3 а м е ч а н и е 9.1. Нулевое значение $P_{-}$в представлении

$$
\frac{1}{\mathrm{P}_{-}} \mathbb{I}_{(0, T)} \cdot\left\langle\check{X}-\check{X}^{\hat{\tau}}, \mathrm{P}\right\rangle^{\check{\mathbb{Q}}^{\circ} \cdot \check{\mathbb{G}}}
$$

не создает проблемы в силу леммы 2.3 .

3 а м е ч а н и е 9.2. Функция $h$ в теореме 9.1 вычислена в лемме 9.2 с помощью вспомогательного произведения пространств. С помощью этого подхода мы получили результат без требования дополнительной регулярности функции плотности р. Это означает, что во многих ситуациях $h$ может быть вычислена непосредственно на $(\mathbb{Q}, \mathbb{F})$. Например, если $\mathrm{p}_{+}(u)-(\mathbb{Q}, \mathbb{F})$-мартингал для каждого $u \in[0, T)$, как пояснялось в замечании 7.2 , функция $h(u)$ совпадает с $d\langle X, \mathrm{p}(u)\rangle / d\langle X, X\rangle$ на $[u, T)$.

\section{СПИСОК ЛИТЕРАТУРЫ}

1. Aldous D. Weak convergence and the general theory of processes. Berkely: University of California Press, 1981, 148 p. 
2. Barlow M., Emery M., Knight F., Song S., Yor M. Antour d'un théorḿe de Tsirelson sur des filtrations browniennes et non browniennes. Séminaire de Probab., 1998, v. XXXII, p. 264-305.

3. Bielecki T., Crepey S. Counterparty Risk and Funding: A Tale of Two Puzzles. London: Chapman \& Hall/CRC Press, 2014, 338 p.

4. Bielecki T., Rutkowski M. Credit Risk: Modeling, Valuation and Hedging. Berlin, New York: Springer, 2002, 540 p.

5. Bielecki T., Jeanblanc M., Rutkowski M. Credit Risk. Lisbonn: Lecture in Cycle of Advanced Courses on Mathematical Finance, 2006.

6. Bielecki T., Jeanblanc M., Rutkowski M. Credit Risk Modelling. Osaka: Osaka University Press, 2009.

7. Dellacherie C., Meyer P. Probabilités et potentiel. Chap. I-IV. Paris: Hermann, 1975, $476 \mathrm{p}$.

8. He S. W., Wang J. G., Yan J.A. Semimartingale Theory and Stochastic Calculus. London: Science Press/CRC Press Inc., 1992, 400 p.

9. Jacod J. Calcul Stochastique et Problémes de Martingales. Lecture Notes in Mathematics, v. 714. Berlin: Springer, 1979, 539 p.

10. Jeanblanc M., LeCam Y. Progressive enlargement of filtration with initial times. Stochastic Process. Appl., 2009, v. 119, № 8, p. 2523-2543.

11. Jeanblanc $M$., Song $S$. An explicit model of default time with given survival probability. — Stochastic Process. Appl., 2010, v. 121, № 8, p. 1678-1704.

12. Jeanblanc $M$., Song $S$. Random times with given survival probability and their $\mathbb{F}$ martingale decomposition formula. - Stochastic Process. Appl., 2010, v. 121, №6, p. $1389-1410$.

13. Jeulin T. Semi-martingales et grossissement d'une filtration. Lecture Notes in Math. 833. Berlin: Springer, 1980, 142 p.

14. Jeulin T., Yor M. Nouveaux résultats sur le grossissement des tribus. - Ann. Scient. Ec. Norm. Sup., 1978, v. 11, № 4, p. 429-443.

15. Jeulin T., Yor M. Grossissement d'une filtration et semi-martingales: formules explicites. - Séminaire Probab., 1978, v. 12, p. 78-97.

16. Li L., Rutkowski M. Progressive enlargement of filtration with pseudo-honest times. Ann. Appl. Probab., 2014, v. 24, № 4, p. 1509-1553.

17. Nelsen R. An Introduction to Copulas. Berlin: Springer, 2006, 272 p.

18. Protter P. Stochastic Integration and Differential Equations. Berlin: Springer, 2005, $415 \mathrm{p}$.

19. Rogers L., Williams D. Diffusions, Markov Processes, and Martingales, Vol. 1: Foundations. Chichester: John Wiley, 1994, 386 p.

20. Song S. Grossissement d'une filtration et problumes connexes. - Thesis Université Paris VI, 1987.

21. Song S. Optional splitting formula in a progressively enlarged filtration. - ESAIM Probability and Statistics, 2013, v. 18, p. 829-853.

22. Song S. Local solution method for the problem of enlargement of filtration, 2013, arxiv: 1302.2862 .

23. Song S. Dynamic one-default model. Arbitrage, Credit and Informational Risks. Proceedings of the Sino-French Research Program in Financial Mathematics Conference. Eds.: Hillairet C., Jeanblanc M., Jiao Y. Singapore: World Scientific Publishing Company, 2013, p. 119-146.

24. Stricker C., Yor M. Calcul stochastique dépendant d'un paramétre. - Z. Wahrsch. verw. Geb., 1978, v. 45, p. 109-133.

25. $W u$ D. Dynamized copulas and applications to counterparty credit risk. - Thesis University Evry Val d'Essonne, 2013.

Поступила в редакцию 31.XII.2014

Исправленный вариант 19.IX.2015 